\title{
Are seamounts refuge areas for fauna from polymetallic nodule fields?
}

\author{
Daphne Cuvelier $^{1 \star \star}$, Pedro A. Ribeiro ${ }^{1, \mathrm{a}, \star}$, Sofia P. Ramalho ${ }^{1, \mathrm{~b}, \star}$, Daniel Kersken ${ }^{2,3}$, Pedro Martinez Arbizu $^{3}$, and \\ Ana Colaço ${ }^{1}$ \\ ${ }^{1}$ Marine and Environmental Sciences Centre (MARE), Instituto do Mar (IMAR), Centro Okeanos, Universidade dos Açores, \\ Rua Prof. Dr. Frederico Machado 4, 9901-862 Horta, Portugal \\ ${ }^{2}$ Department of Marine Zoology, Senckenberg Research Institute and Natural History Museum, Senckenberganlage 25, \\ 60325 Frankfurt am Main, Germany \\ ${ }^{3}$ German Centre for Marine Biodiversity Research (DZMB), Senckenberg am Meer, Südstrand 44, \\ 26382 Wilhelmshaven, Germany \\ ${ }^{a}$ current address: Department of Biological Sciences and K.G. Jebsen Centre for Deep Sea Research, \\ University of Bergen, Bergen, Norway \\ b current address: Centro de Estudos do Ambiente e do Mar (CESAM), Departmento de Biologia, \\ Universidade de Aveiro, Campus Universitário de Santiago, 3810-193 Aveiro, Portugal \\ These authors contributed equally to this work.
}

Correspondence: Daphne Cuvelier (daphne.cuvelier@gmail.com)

Received: 6 August 2019 - Discussion started: 12 August 2019

Revised: 9 March 2020 - Accepted: 23 March 2020 - Published: 15 May 2020

\begin{abstract}
Seamounts are abundant and prominent features on the deep-sea floor and intersperse with the nodule fields of the Clarion-Clipperton Fracture Zone (CCZ). There is a particular interest in characterising the fauna inhabiting seamounts in the CCZ because they are the only other ecosystem in the region to provide hard substrata besides the abundant nodules on the soft-sediment abyssal plains. It has been hypothesised that seamounts could provide refuge for organisms during deep-sea mining actions or that they could play a role in the (re-)colonisation of the disturbed nodule fields. This hypothesis is tested by analysing video transects in both ecosystems, assessing megafauna composition and abundance.

Nine video transects (ROV dives) from two different license areas and one Area of Particular Environmental Interest in the eastern CCZ were analysed. Four of these transects were carried out as exploratory dives on four different seamounts in order to gain first insights into megafauna composition. The five other dives were carried out in the neighbouring nodule fields in the same areas. Variation in community composition observed among and along the video transects was high, with little morphospecies overlap along intra-
\end{abstract}

ecosystem transects. Despite the observation of considerable faunal variations within each ecosystem, differences between seamounts and nodule fields prevailed, showing significantly different species associations characterising them, thus calling into question their use as a possible refuge area.

\section{Introduction}

Seamounts are abundant and prominent features on the deep-sea floor (Wessel et al., 2010). They are common in all the world's oceans, occurring in higher abundances around mid-ocean ridges, island-arc convergent areas and above upwelling mantle plumes (Kitchingman et al., 2007). Seamounts are defined as isolated subsurface topographic features, usually of volcanic origin and of significant height above the seafloor (International Seabed Authority (ISA), 2019). They are generally isolated, typically cone-shaped undersea mountains rising relatively steeply at least several hundred metres from the deep-sea floor. Seamounts comprise a unique deep-sea environment, characterised by substantially enhanced currents and fauna that is dominated by 
suspension feeders, such as corals (Rogers, 2018). They represent hard substrata in the otherwise soft-sediment deep sea and can thus be considered habitat islands (Beaulieu, 2001). Given the growing evidence that seamounts differ substantially across a range of spatial scales, the concept of seamounts as a single, relatively well-defined habitat type is outdated (Clark et al., 2012). Depth and substrate type are key elements in determining the composition and distribution of benthic fauna on seamounts, while location is likely the subsequent most important driver of faunal composition and distribution patterns (e.g. Tittensor et al., 2009). Connectivity varies substantially between seamounts, resulting in the presence of taxa with very localised to very wide distributions (Clark et al., 2010).

The Clarion-Clipperton Fracture Zone (CCZ), in the equatorial eastern Pacific Ocean, is most known for its extensive polymetallic nodule fields that will potentially be mined in the future. In this area, nodules represent the most common hard substrate on the soft-sediment abyssal plains, and many organisms rely on them for survival (Vanreusel et al., 2016). Removal of hard substrate through mining actions will impact all these organisms, which were estimated at about $50 \%$ of all megafaunal species in the CCZ (Amon et al., 2016). Nodule fields in the CCZ are interspersed by seamounts (Wedding et al., 2013), the only feature offering hard substrata besides the nodules. Based on this feature or characteristic, it has been hypothesised that seamounts could provide refuge for organisms during deep-sea mining activities or that seamounts could play a role in the (re-)colonisation of the disturbed nodule fields. Whether or not this is true may have important implications for the management of the impacts of polymetallic nodule mining in the CCZ. However, knowledge of the biodiversity inhabiting seamounts in this region is currently lacking.

The objectives of the current study were twofold: (i) provide first insights into seamount megafauna within the CCZ and (ii) compare the benthic fauna inhabiting seamounts and nodule fields in the eastern CCZ. Since this is the first time the seamounts in the eastern $\mathrm{CCZ}$ were visited, a separate section is dedicated to describing these first insights.

\section{Material and methods}

\subsection{Study site and data}

During the SO239 EcoResponse cruise in 2015 (Martinez Arbizu and Haeckel, 2015), four seamounts were visited for the first time within two different license areas and one Area of Particular Environmental Interest (APEI) within the Clarion-Clipperton Fracture Zone (CCZ; Table 1). Nodule fields within the same license areas were visited and sampled as well. Video imagery and faunal samples were collected by a remotely operated vehicle (ROV KIEL 6000 (GEOMAR), equipped with a high-definition Kongsberg OE14-500 camera).

Seamount transects were carried out uphill, towards the summit, resulting in a depth gradient along the transect (Table 1). The four seamount transects were characterised by different depth ranges and lengths and were, due to the vessel's positioning and the predominant south-east surface currents, all carried out downstream, on the north to northwestern flanks of the seamounts (Table 1 and Fig. 1). The names of the seamounts used here, Rüppel and Senckenberg (BGR; German License area), Heip (GSR; Belgian License area) and Mann Borgese (APEI3), are the ones agreed upon by the scientist during the EcoResponse cruise (Martinez Arbizu and Haeckel, 2015), pending incorporation of these names into the GEBCO gazetteer. The seamounts differed in shape and size with Senckenberg and Heip being sea-mountain ranges, while Rüppel and Mann Borgese were more isolated, stand-alone seamounts (Fig. 1). Nodule field dives were carried out on relatively flat surfaces (maximum depth range covered during a dive or transect was $30 \mathrm{~m}$ difference; Table 1) and were referred to by the dive number and license area. The five nodule transects were all located between 4000 and $5000 \mathrm{~m}$ depth, and the transects differed in length between dives as well (Table 1). Within the same license area, the distance between different transects was 16 to $60 \mathrm{~km}$, while distance between license areas added up to several hundreds of kilometres (minimum $\sim 700 \mathrm{~km}$ BGRGSR; Fig. 1).

Investigated areas were restricted to the eastern part of the CCZ with APEI3 being the most northward- and westwardbound area. The optical resolution of the camera enabled reliable identification of organisms larger than $3 \mathrm{~cm}$ (Martinez Arbizu and Haeckel, 2015). The combination of exploration and opportunistic sampling restricted a systematic image collection. The target ROV travelling altitude was $<2 \mathrm{~m}$, and travelling speed was $\sim 0.2 \mathrm{~m} \mathrm{~s}^{-1}$; both altitude and speed, along with the camera zoom, were kept constant whenever possible.

\subsection{Video analysis and statistics}

All videos were annotated to the lowest taxonomic level possible. The number of morphospecies, defined as morphologically different organisms within the lowest taxonomic group identified, were assessed. Identifications were doublechecked with scientists working in the same area as well as taxonomic experts and comprise different taxonomic levels (e.g. genus, family), and organism samples were used for proper identification whenever possible. Those identifications restricted to higher taxon groups (family, class, etc.) and for which it was impossible to attribute a morphospecies, were referred to as taxa and are likely to morphologically differ between transects. Xenophyophores, living on the softsediment deep-sea floor, were less prominently present on seamounts than in nodule fields and were not quantified. Fish 
Table 1. Overview giving details of imagery transects analysed in the Clarion-Clipperton license areas. Video duration includes time spent sampling. Number of observations includes undetermined organisms. Transect lengths do not include parts visualising ancient disturbance tracks or parts when the seafloor was not visualised or visible.

\begin{tabular}{lllrrrr}
\hline Station and dive & $\begin{array}{l}\text { License } \\
\text { area }\end{array}$ & $\begin{array}{l}\text { Seamount }(\mathrm{SM}) \\
\text { or nodule field (NF) }\end{array}$ & $\begin{array}{r}\text { Depth } \\
(\mathrm{m})\end{array}$ & $\begin{array}{r}\text { Transect } \\
\text { length }\end{array}$ & $\begin{array}{r}\text { Approximate surface } \\
\text { covered }\left(\mathrm{m}^{2}\right)\end{array}$ & $\begin{array}{r}\text { Faunal densities } \\
\text { (ind. 100 m }\end{array}$ \\
\hline SO239_29_ROV02 & BGR & SM Rüppell & $3000-2500$ & $1250 \mathrm{~m}$ & 9458.6 & 4.4 \\
SO239_41_ROV03 & BGR & NF & $4080-4110$ & $1590 \mathrm{~m}$ & 5309.1 & 19.3 \\
SO239_54_ROV04 & BGR & SM Senckenberg & $3350-2850$ & $2500 \mathrm{~m}$ & 12288.5 & 6.9 \\
SO239_131_ROV08 & GSR & NF & $4470-4480$ & $710 \mathrm{~m}$ & 1602.5 & 30.3 \\
SO239_135_ROV09 & GSR & SM Heip & $3900-3550$ & $1000 \mathrm{~m}$ & 6905.4 & 5.3 \\
SO239_141_ROV10 & GSR & NF & $4455-4480$ & $520 \mathrm{~m}$ & 1683.4 & 27.6 \\
SO239_189_ROV13 & APEI 3 & NF & $4890-4930$ & $1790 \mathrm{~m}$ & 3580.0 & 3.8 \\
SO239_200_ROV14 & APEI 3 & NF & $4650-4670$ & $1490 \mathrm{~m}$ & 2980.0 & 6.2 \\
SO239_212_ROV15 & APEI 3 & SM Mann Borgese & $1850-1650$ & $900 \mathrm{~m}$ & 4805.3 & 7.6 \\
\hline
\end{tabular}

(Actinopterygii), Crustacea (Nematocarcinidae, Aristeidae, Peracarida) and Polychaeta were quantified but left out of the comparative statistical analysis due to their lack of representativity and being possibly attracted due to the ROV lights. The same applied to jellyfish and other doubtful identifications that could not be confidently assigned to a higher taxonomic group (Table 2). A subset of the nodule field transects from BGR, GSR and APEI3 was presented by Vanreusel et al. (2016), corresponding to $44 \%$ of what was studied here, and limited organism identification to a higher taxonomic level (order, e.g. Alcyonacea, or class, e.g. Ophiuroidea). In our study, all the transects $(100 \%)$ were annotated to morphospecies level, allowing a detailed comparison between seamounts and nodule fields.

Three categories of substratum types were distinguished - (1) predominant soft substrata $(<40 \%$ hard substrata $)$, (2) mix or transition (between 40 and $60 \%$ hard substrata) and (3) predominant hard substrata ( $>60 \%$ hard substrata) and were annotated at $10 \mathrm{~m}$ distance units based on the video footage and tested for correlations with taxonomic abundances.

ROV transects along the seamounts were carried out as exploratory dives. The sampling strategy both on seamounts and in nodule fields combined video and sampling or specimen collection. The travelling altitude was more easily maintained along the relatively flat nodule field transects, where an average of $93 \%$ of the time was spent at altitudes of $<$ $2 \mathrm{~m}$. Contrastingly, the uphill seamount transects were more variable in ROV altitude, with on average $61 \%$ of the time spent at $<2 \mathrm{~m}$ altitudes and the remaining $\sim 39 \%$ spent at higher altitudes, which generally resulted in a higher surface covered on the seamounts. Approximate surface covered $\left(\mathrm{m}^{2}\right)$ was then estimated by using the ROV altitude, time spent at a predefined altitude, distance travelled and the image widths at predefined altitudes. The following altitude ranges (and image widths, following Vanreusel et al. (2016) and extrapolated thereon) were taken into account: $<1 \mathrm{~m}$ (2 m), 1-2 m (4 m), 2-3 m (6 m), 3-4 m (8m) and 4-5 m
$(10 \mathrm{~m})$. Altitude ranges of $>5 \mathrm{~m}$, adding up to $12 \%$ for seamount transects and $3 \%$ for nodule field transects, were left out since these were the parts where the seafloor was not visualised or organisms could not be quantified. Due to the explorative nature of the dives, the pan and tilt of the ROV camera were not kept constant and thus represent a bias in the surface estimations. Visualisation of ancient disturbance tracks were omitted as well, as these fell out of the scope of the article. Faunal densities were calculated as individuals per hundred square metres (ind. $100 \mathrm{~m}^{-2}$ ). Statistical testing was carried out in R (R Core Team, 2018). Nonmetric multidimensional scaling analysis (nMDS) was based on the Bray-Curtis dissimilarity and carried out with the vegan package (Oksanen et al., 2018). Kendall's coefficient of concordance $(W)$ was calculated to identify significantly associated groups of species, based on correlations and permutations (Legendre, 2005).

\section{Results}

About $80 \%$ of all taxa observed across the two adjacent ecosystems could be identified to a morphospecies level. On first view, morphospecies were revealed to be quite different between seamounts and nodule fields (Fig. 2). While the number of faunal observations along the seamount transects were within similar ranges (4.4-7.6 ind. $100 \mathrm{~m}^{-2}$ ), those along the nodule transects featured both the highest and lowest values (3.8-30.3 ind. $100 \mathrm{~m}^{-2}$; Table 1). The lowest number of faunal observations was performed along the two APEI3 nodule transects (ROV13 and ROV14) and the highest along the GSR nodule transect ROV08. What follows is a first description of eastern CCZ seamount megafauna (Sect. 3.1.) and a detailed comparison with the neighbouring nodule fields (Sect. 3.2.) 

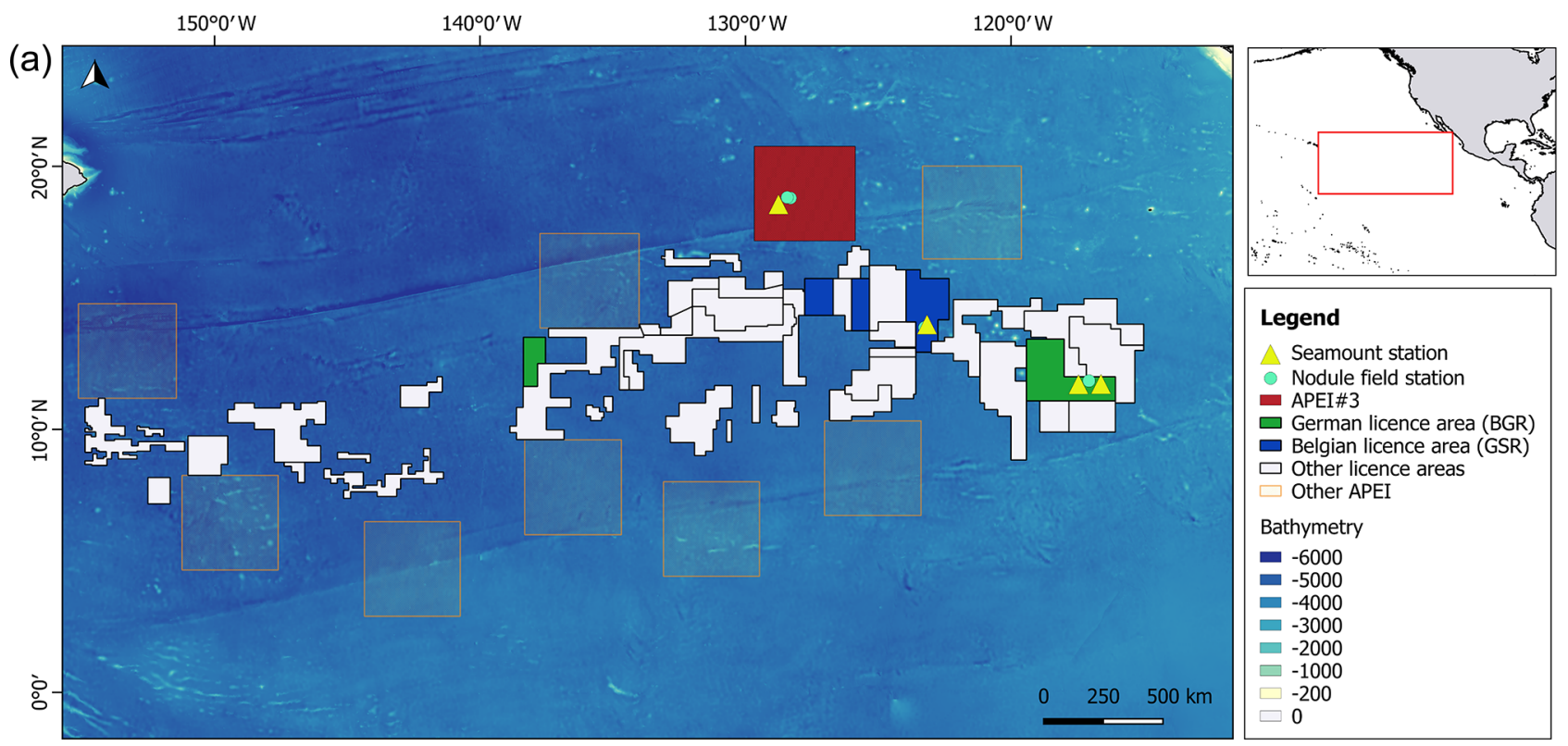

(b) Mann Borgese (APEI3)
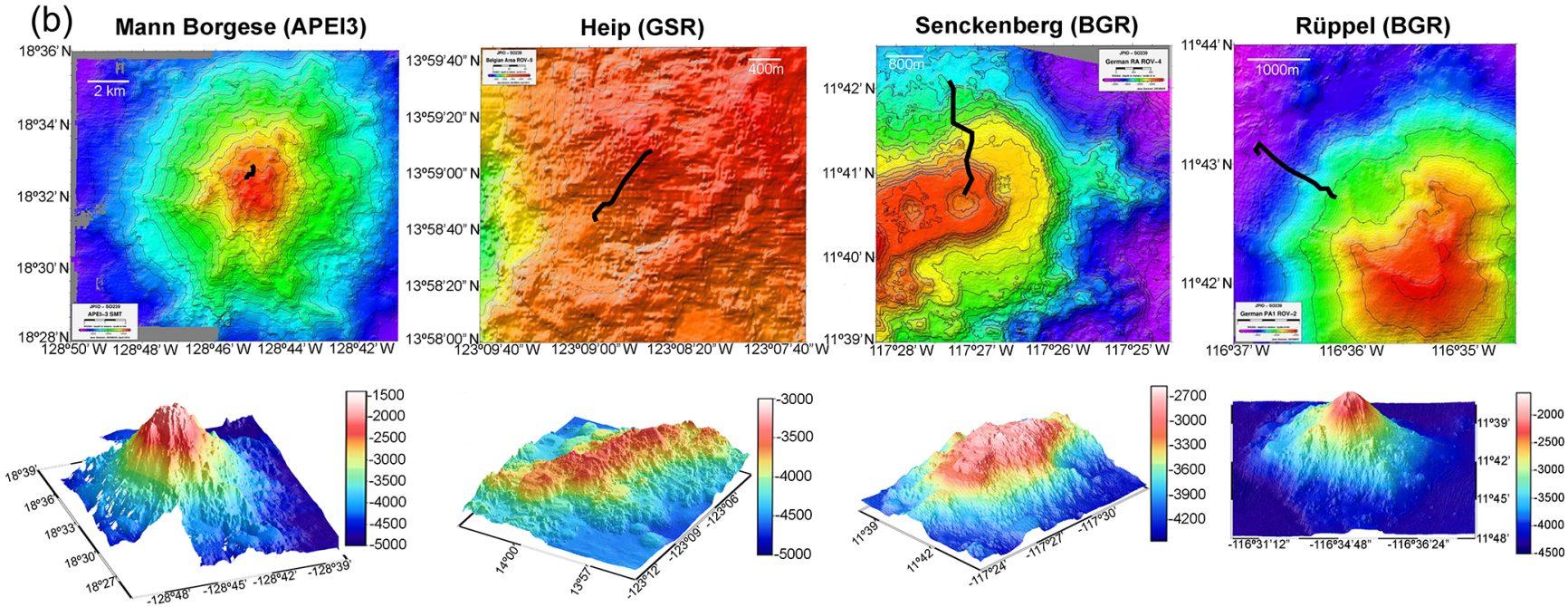

Figure 1. (a) Location of the Clarion-Clipperton Fracture Zone in the equatorial eastern Pacific Ocean featuring the contract areas of the International Seabed Authority (ISA) and the positions of the sampled areas (seamounts and nodule fields). Information on transect length and depth gradients can be found in Table 1. (b) Location of the seamount transects carried out towards the summit on the north-northwestern flank and seamount profiles. Rüppel (BGR; ROV02) and Mann Borgese (APEI3; ROV15) are single seamounts, while Senckenberg (BGR; ROV04) and Heip (GSR; ROV09) are sea-mountain ranges. Bathymetry is given in metres.

\subsection{Insights into CCZ seamount megafauna}

The most abundant and diverse (most morphospecies) taxa along the seamount transects comprised Echinodermata (Asteroidea, Crinoidea, Holothuroidea, Ophiuroidea), Anthozoa (Actiniaria, Alcyonacea, Pennatulacea) and Porifera (Hexactinellida); Table 2, Figs. 3, A1 in the Appendix). Keeping in mind the limitation of the video sampling, differences among the benthic seamount community composition are described here. The transect at Mann Borgese (APEI3) was characterised by high densities of Antipatharia, more specifically Antipathidae (3.5 ind. $100 \mathrm{~m}^{-2}$ ), and solitary Sclerac- tinia (1.5 ind. $100 \mathrm{~m}^{-2}$; Table 2, Fig. A1). Antipathidae observations were mostly grouped at the end of the video transect, i.e. at the summit. Densities of both Antipatharia and Scleractinia were much lower along the other seamount transects $\left(<0.2\right.$ ind. $\left.100 \mathrm{~m}^{-2}\right)$, with Scleractinia being absent from the Heip and Senckenberg transects. Alcyonacea corals were observed along all seamount transects. Isididae were found along the Senckenberg and Heip transects, and one individual from the Chrysogorgiidae family was observed along the latter as well. Varying numbers of Primnoidae were observed along all transects (Table 2). High abundances of Pennatulacea were observed at Senckenberg 


\section{Seamounts}
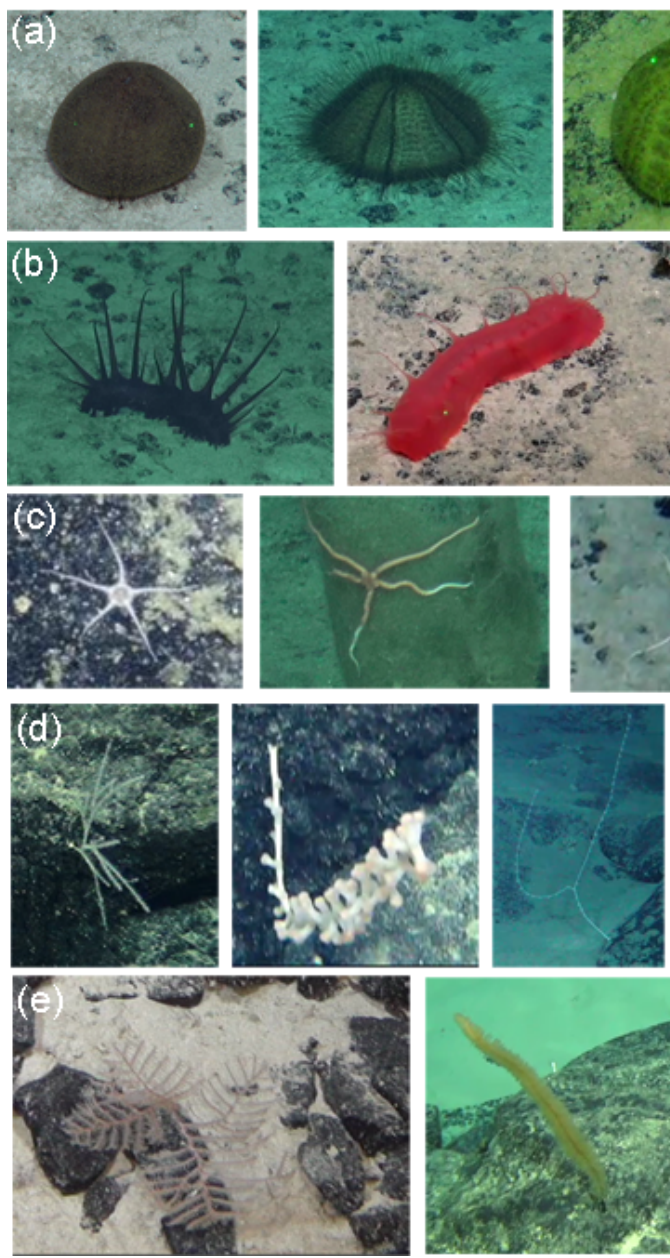
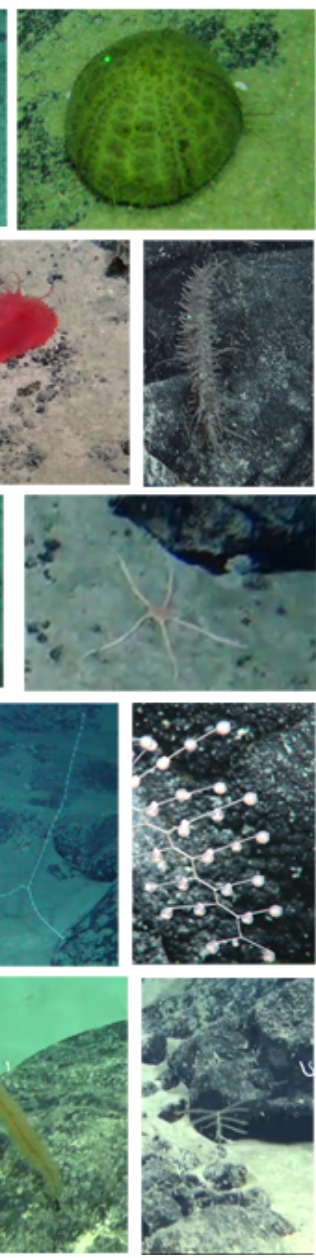

Nodule fields
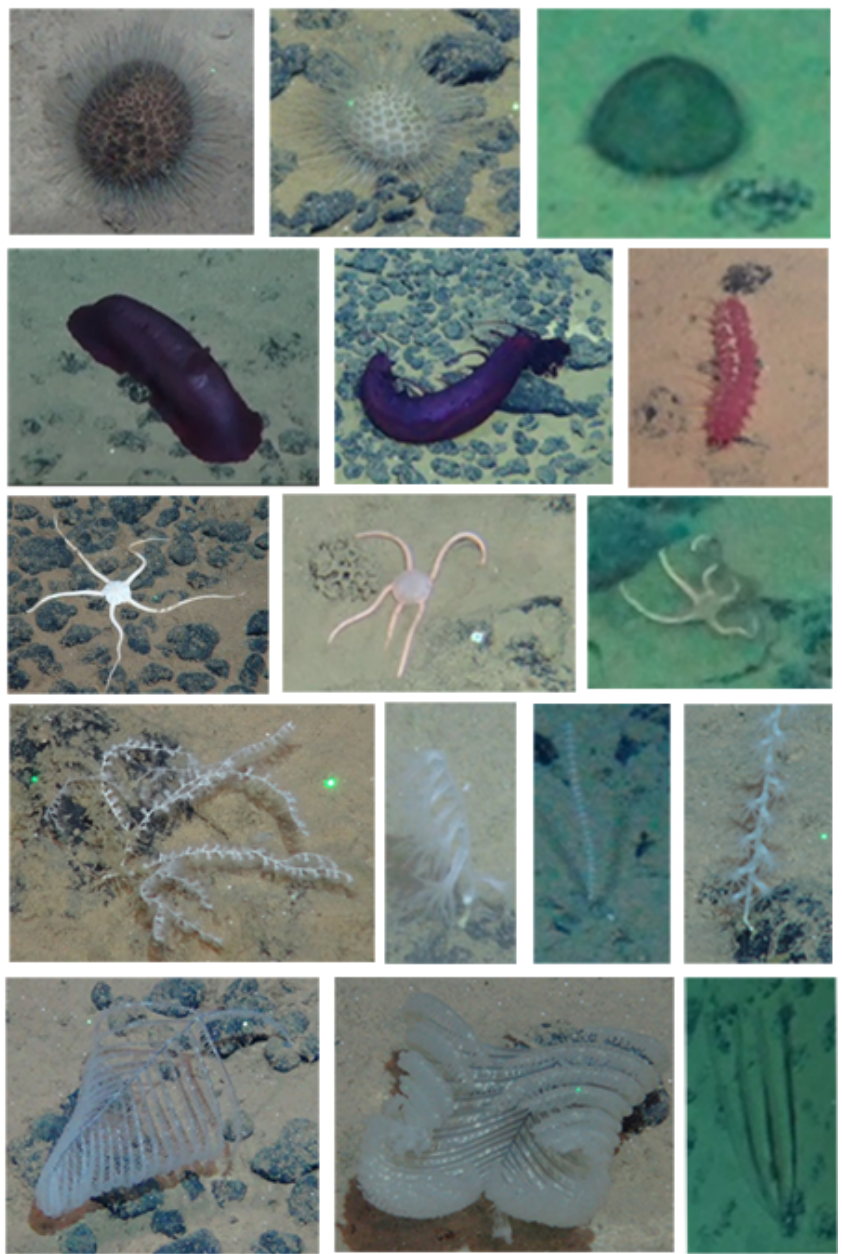

Figure 2. Some examples of different morphospecies (msp.) on seamounts and in nodule fields in the CCZ. Selected taxa were (a) Echinoidea (from left to right: Urechinidae msp. 4 (URC_019), Urechinidae msp. 2 (URC_013), Urechinidae msp. 3 (URC_009), Urechinidae msp. A (URC_020), Urechinidae msp. B (URC_021), Urechinidae msp. C (URC_005)), (b) Holothuroidea (from left to right: Psychropotidae msp. 1 (HOL_088), Benthodytes red msp. (HOL_101), Deimatidae - irregular papillae - msp. (HOL_070), Psychropotes verrucosa (HOL_045), Laetmogonidae (HOL_030), Synallactes msp. 2 pink (HOL_008)), (c) Ophiuroidea (from left to right: Ophiuroidea msp. 5 (OPH_003), Ophiuroidea msp. 4 (OPH_005), Ophiuroidea msp. 6 (OPH_006), Ophiuroidea msp. 6 (OPH_006), Ophiuroidea (OPH_012), Ophiuroidea msp. 4 (OPH_005)), (d) Alcyonacea (from left to right: Callozostron cf. bayeri (ALC_009), Bathygorgia aff. profunda 2 (ALC_005), Keratoisis aff. flexibilis msp. 2 (ALC_029), Chrysogorgia cf. pinnata, Abyssoprimnoa cf. gemina (ALC_008), Bathygorgia aff. profunda 1, Calyptrophora cf. persephone (ALC_007), Bathygorgia aff. abyssicola 1 (ALC_003), ) and (e) Antipatharia (from left to right: Umbellapathes aff. helioanthes (ANT_018), cf. Parantipathes morphotype 1 (ANT_017), Bathypathes cf. alternata msp. 1 (ANT_010), Bathypathes cf. alternata (ANT_006), Abyssopathes cf. lyra (ANT_022), Bathypathes sp. (ANT_003)). Codes refer to an ongoing collaboration in creating one species catalogue for the CCZ and align all morphospecies of different research groups. Copyright: SO239, ROV KIEL 6000, GEOMAR Helmholtz Centre for Ocean Research Kiel.

(0.7 ind. $\left.100 \mathrm{~m}^{-2}\right)$, representing about $20 \%$ of sessile fauna annotations for this transect.

Enteropneusta were only observed along the Rüppel and Senckenberg transects in the BGR area, represented by two different morphospecies, namely Yoda morphospecies (Torquaratoridae) at Rüppel and Saxipendium morphospecies (Harrimaniidae) at Senckenberg.

The highest Polychaeta densities were observed along the Heip transect in the GSR area, which was mainly due to high densities of free-swimming Acrocirridae $\left(0.5\right.$ ind $100 \mathrm{~m}^{-2}$ vs. $0.02-0.03$ ind $100 \mathrm{~m}^{-2}$ in BGR area; Table 2). Aphroditidae polychaetes were only present along the BGR transects ( 0.02 ind. $100 \mathrm{~m}^{-2}$, corresponding to three individuals along the transect at Rüppel and one individual along the transect at Senckenberg; Table 2).

Porifera densities were highest along the Heip transect $\left(0.93\right.$ ind. $\left.100 \mathrm{~m}^{-2}\right)$, followed by Senckenberg $\left(0.38\right.$ ind. $\left.100 \mathrm{~m}^{-2}\right)$, Mann Borgese $\left(0.36\right.$ ind. $\left.100 \mathrm{~m}^{-2}\right)$ 
Table 2. Overview of all densities (ind. $100 \mathrm{~m}^{-2}$ ) observed within each video transect. Higher taxa are in bold. Indeterminate (indet.) organisms were organisms impossible to attribute to a lower taxonomic group. ROV02 is Rüppel; ROV04 is Senkcenberg; ROV09 is Heip; ROV15 is Mann Borgese; msp. is morphospecies; gen. is genus.

\begin{tabular}{|c|c|c|c|c|c|c|c|c|c|}
\hline & \multicolumn{4}{|c|}{ Seamounts } & \multicolumn{5}{|c|}{ Nodule fields } \\
\hline & ROV2 & ROV4 & ROV9 & ROV15 & ROV3 & ROV8 & ROV10 & ROV13 & ROV14 \\
\hline & ind. $100 \mathrm{~m}^{-2}$ & ind. $100 \mathrm{~m}^{-2}$ & ind. $100 \mathrm{~m}^{-2}$ & ind. $100 \mathrm{~m}^{-2}$ & ind. $100 \mathrm{~m}^{-2}$ & ind. $100 \mathrm{~m}^{-2}$ & ind. $100 \mathrm{~m}^{-2}$ & ind. $100 \mathrm{~m}^{-2}$ & ind. $100 \mathrm{~m}^{-2}$ \\
\hline \multicolumn{10}{|l|}{ Annelida* } \\
\hline Polychaeta indet.* (No Serpulidae) & 0.02 & 0.02 & & 0.02 & 0.09 & & 0.12 & 0.03 & 0.03 \\
\hline Acrocirridae & 0.02 & 0.03 & 0.52 & & 0.17 & 0.06 & 0.18 & 0.89 & 0.97 \\
\hline Aphroditidae & 0.03 & 0.01 & & & & & & & \\
\hline Echiura msp. 1 & & & & & 0.17 & 0.50 & 0.36 & & 0.10 \\
\hline \multicolumn{10}{|l|}{ Polynoidae } \\
\hline Polynoidae msp. 2 & & & & & & 0.06 & 0.18 & & \\
\hline Polynoidae white msp. & & & & & & 0.06 & & 0.03 & 0.07 \\
\hline \multicolumn{10}{|l|}{ Bryozoa } \\
\hline Bryozoa msp. 2 & & & 0.02 & & & & & & \\
\hline Bryozoa indet. & & 0.01 & & & 0.13 & 0.69 & 0.06 & 0.06 & 0.07 \\
\hline \multicolumn{10}{|l|}{ Cnidaria } \\
\hline \multicolumn{10}{|l|}{ Anthozoa } \\
\hline \multicolumn{10}{|l|}{ Ceriantharia } \\
\hline Ceriantharia msp. 1 & 0.04 & 0.01 & 0.05 & 0.04 & & 0.06 & & & \\
\hline Ceriantharia msp. 2 & & & & 0.08 & & 0.12 & 0.06 & & \\
\hline Ceriantharia indet. & & & 0.01 & & & & & & \\
\hline \multicolumn{10}{|l|}{ Hexacorallia } \\
\hline \multicolumn{10}{|l|}{ Actiniaria } \\
\hline Actinoscyphiidae & & 0.02 & & & & & & & \\
\hline Actiniidae Bolocera msp. & 0.13 & 0.04 & & & & & & & \\
\hline Actiniaria msp. 15 & 0.01 & & & & & & & & \\
\hline Actiniaria msp. 4 & & 0.02 & & 0.02 & & & & & \\
\hline Actiniaria msp. 5 & 0.01 & 0.02 & & 0.06 & & & & & \\
\hline Actiniaria msp. 10 & & & & & 0.09 & & & & 0.17 \\
\hline Actiniaria msp. 2 & & & & & 0.32 & 0.50 & 0.06 & & 0.13 \\
\hline Actiniaria msp. C & & & & & 0.11 & 0.19 & 0.12 & & \\
\hline Actiniaria msp. D & & & & & 0.02 & & & & \\
\hline Actiniaria msp. 7 & & & & & 0.19 & 0.06 & 0.18 & 0.03 & 0.07 \\
\hline Actiniaria msp. 8 & & & & & 0.04 & 1.62 & 0.95 & & 0.10 \\
\hline Actiniaria msp. 9 & & & & & & & 0.06 & & \\
\hline Actiniaria msp. A & & & & & & & 0.06 & & 0.03 \\
\hline Actiniaria msp. B & & & & & 0.08 & 0.06 & 0.12 & 0.03 & \\
\hline Actiniaria indet. & 0.02 & 0.03 & & & 0.47 & 0.62 & 1.37 & 0.06 & 0.07 \\
\hline \multicolumn{10}{|l|}{ Antipatharia } \\
\hline \multicolumn{10}{|l|}{ Antipathidae } \\
\hline Antipathes msp. 1 & & & & 1.59 & & & & & \\
\hline Antipathes msp. 2 & & & & 0.02 & & & & & \\
\hline Stichopathes msp. 1 & & & & 1.75 & & & & & \\
\hline Antipathidae indet. & & & & 0.10 & & & & & \\
\hline \multicolumn{10}{|l|}{ Schizopathidae } \\
\hline Abyssopathes cf. lyra & & & & & 0.15 & 0.25 & 0.18 & & \\
\hline Bathypathes cf. alternata & & & & & & 0.06 & 0.06 & & 0.03 \\
\hline Bathypathes $\mathrm{cf}$. alternata $\mathrm{msp} .1$ & & & 0.01 & & & & & & \\
\hline Bathypathes cf. alternata msp. 2 & & 0.02 & & & & & & & \\
\hline Bathypathes sp. & & & & & 0.06 & 0.06 & & & \\
\hline Bathypathes msp. 1 & & & 0.01 & & & & & & \\
\hline cf. Parantipathes msp. 1 & & & 0.02 & & & & & & \\
\hline Umbellapathes aff. bipinnata & & 0.04 & 0.01 & & & & & & \\
\hline Umbellapathes aff. helioanthes & & 0.12 & & & & & & & \\
\hline Antipatharia indet. & 0.01 & 0.02 & 0.01 & 0.12 & 0.08 & 0.12 & 0.06 & & \\
\hline
\end{tabular}


Table 2. Continued.

\begin{tabular}{|c|c|c|c|c|c|c|c|c|c|}
\hline & \multicolumn{4}{|c|}{ Seamounts } & \multicolumn{5}{|c|}{ Nodule fields } \\
\hline & ROV2 & ROV4 & ROV9 & ROV15 & ROV3 & ROV8 & ROV10 & ROV13 & ROV14 \\
\hline & ind. $100 \mathrm{~m}^{-2}$ & ind. $100 \mathrm{~m}^{-2}$ & ind. $100 \mathrm{~m}^{-2}$ & ind. $100 \mathrm{~m}^{-2}$ & ind. $100 \mathrm{~m}^{-2}$ & ind. $100 \mathrm{~m}^{-2}$ & ind. $100 \mathrm{~m}^{-2}$ & ind. $100 \mathrm{~m}^{-2}$ & ind. $100 \mathrm{~m}^{-2}$ \\
\hline \multicolumn{10}{|c|}{ Corallimorpharia/Corallimorphidae } \\
\hline Corallimorphus msp. 1 & & 0.01 & & & & & & & \\
\hline Corallimorphus msp. 2 & & 0.09 & 0.01 & & 0.08 & 0.12 & 0.06 & & \\
\hline Corallimorpharia msp. 3 & & 0.01 & & & & & & & \\
\hline Corallimorpharia msp. 4 & & & 0.01 & & & & & & \\
\hline Corallimorpharia msp. A & & & & & 0.02 & & 0.06 & & \\
\hline \multirow{2}{*}{\multicolumn{10}{|c|}{ Scleractinia }} \\
\hline & & & & & & & & & \\
\hline Scleractinia msp. 1 & 0.02 & & & 1.47 & & & & & \\
\hline \multicolumn{10}{|l|}{ Zoantharia } \\
\hline Zoantharia msp. 2 & & & & 0.02 & & & & & \\
\hline Zoantharia indet. & & 0.09 & & 0.04 & & & & & \\
\hline \multirow{2}{*}{\multicolumn{10}{|c|}{$\begin{array}{l}\text { Octocorallia } \\
\text { Alcyonacea }\end{array}$}} \\
\hline & & & & & & & & & \\
\hline \multicolumn{10}{|l|}{ Alcyoniidae } \\
\hline Anthomastus msp. 1 & 0.03 & & & & & & & & \\
\hline Anthomastus msp. 2 & 0.00 & 0.03 & & 0.02 & & & & & \\
\hline \multicolumn{10}{|l|}{ Coralliidae } \\
\hline Corallium sp. nov. & & & & 0.02 & & & & & \\
\hline \multicolumn{10}{|l|}{ Chrysogorgiidae } \\
\hline Chrysogorgia cf. pinnata & & & 0.01 & & & & & & \\
\hline \multicolumn{10}{|l|}{ Isididae } \\
\hline Bathygorgia aff. abyssicola 1 & & & & & & 0.06 & 0.06 & & \\
\hline Bathygorgia aff. profunda 1 & & 0.03 & 0.01 & & & & & & \\
\hline Bathygorgia aff. profunda 2 & & & 0.01 & & & & & & \\
\hline Keratoisis aff. flexibilis msp. 2 & & & 0.01 & & & & & & \\
\hline Isididae msp. 1 & & 0.01 & & & & & & & \\
\hline \multirow{2}{*}{\multicolumn{10}{|c|}{ Taiaroidae }} \\
\hline & & & & & & & & & \\
\hline \multirow{2}{*}{\multicolumn{10}{|c|}{ Primnoidae }} \\
\hline & & & & & & & & & \\
\hline Abyssoprimnoa cf. gemina & & & & & & 0.31 & 0.18 & & \\
\hline Callozostron cf. bayeri & 0.01 & 0.11 & & & 0.02 & & & & \\
\hline Calyptrophora cf. persephone & & & & & 0.02 & & & & \\
\hline Narella msp. 1 & & 0.02 & & 0.02 & & & & & \\
\hline Primnoidae indet. & 0.08 & & 0.02 & & 0.81 & 1.50 & 0.48 & & \\
\hline Alcyonacea msp. 1 & & & & & 0.04 & & & 0.06 & 0.07 \\
\hline \multirow{2}{*}{\multicolumn{10}{|c|}{ Pennatulacea }} \\
\hline & & & & & & & & & \\
\hline \multicolumn{10}{|l|}{ Umbellulidae } \\
\hline Umbellula msp. 1_white & & & & & & & & & 0.07 \\
\hline Umbellula msp. 1_orange & & 0.06 & & 0.02 & & & & & \\
\hline Umbellula msp. 2 & & 0.02 & & & & & & & \\
\hline Umbellulidae indet. & & 0.03 & & & & & & & \\
\hline Protoptilidae & & & & 0.02 & & & & & \\
\hline Protoptilum msp. 1 & & 0.01 & & 0.04 & & & & & \\
\hline Pennatulacea msp. 2 & & 0.01 & & & & & & & \\
\hline Pennatulacea msp. 5 & & 0.05 & & & & & & & \\
\hline Pennatulacea msp. 6 & & 0.02 & & & & & & & \\
\hline $\begin{array}{l}\text { Pennatulacea msp. } 7 \\
\text { S }\end{array}$ & & 0.08 & & & & & & & \\
\hline Pennatulacea msp. 8 & & 0.02 & & & & & & & \\
\hline Pennatulacea indet. & 0.02 & 0.42 & & 0.02 & & 0.06 & & & \\
\hline Octocorallia msp. 1 & & & & 0.04 & & & & & \\
\hline \multicolumn{10}{|l|}{ Octocorallia msp. 2} \\
\hline Anthozoa indet. & 0.02 & 0.02 & 0.07 & 0.12 & 0.04 & 0.06 & 0.06 & & \\
\hline
\end{tabular}


Table 2. Continued.

\begin{tabular}{|c|c|c|c|c|c|c|c|c|c|}
\hline & \multicolumn{4}{|c|}{ Seamounts } & \multicolumn{5}{|c|}{ Nodule fields } \\
\hline & ROV2 & ROV4 & ROV9 & ROV15 & ROV3 & ROV8 & ROV10 & ROV13 & ROV14 \\
\hline \multicolumn{10}{|l|}{ Hydrozoa } \\
\hline Branchiocerianthus msp. & & 0.02 & & & & & & & \\
\hline Hydrozoa indet. & & 0.02 & 0.01 & 0.04 & & 0.06 & & & \\
\hline \multicolumn{10}{|l|}{ Crustacea* } \\
\hline \multicolumn{10}{|l|}{ Decapoda } \\
\hline Caridea & 0.46 & 0.52 & 0.47 & 0.04 & 0.06 & & 0.36 & 0.06 & 0.10 \\
\hline Decapoda msp. 3 & & 0.02 & & & & & & & \\
\hline Decapoda msp. 4 & 0.01 & & & & & & & & \\
\hline Decapoda Aristeidae & 0.01 & 0.02 & & 0.10 & 0.02 & 0.25 & 0.18 & 0.06 & 0.03 \\
\hline Decapoda msp. 1 & & & & & & & & 0.03 & \\
\hline \multicolumn{10}{|l|}{ Galatheidae } \\
\hline Galatheidae small red msp. & 0.37 & 0.11 & 0.02 & 0.08 & 0.02 & & & & \\
\hline Galatheidae small white msp. & 0.01 & 0.02 & & & & & & & \\
\hline Munidopsis spp. & 0.11 & 0.07 & 0.07 & & & 0.19 & & & 0.10 \\
\hline Galatheidae indet. & 0.02 & 0.03 & 0.02 & 0.02 & & & & & \\
\hline \multicolumn{10}{|l|}{ Parapaguridae } \\
\hline Parapaguridae msp. 1 Probeebei $\mathrm{sp}$. & 0.07 & 0.05 & & & & & & & \\
\hline \multicolumn{10}{|l|}{ Peracarida } \\
\hline Amphipoda & & & 0.01 & & 0.02 & 0.12 & & 0.03 & 0.13 \\
\hline Podoceridae msp. 1 & & & & & & & & 0.03 & \\
\hline Amphipoda msp. 1 & & 0.02 & 0.02 & & & & & & \\
\hline \multicolumn{10}{|l|}{ Isopoda } \\
\hline Munnopsidae msp. 1 & & & & & 0.17 & 0.19 & 0.06 & 0.08 & 0.27 \\
\hline Decapoda indet. & & 0.02 & 0.10 & & & & & & \\
\hline Crustacea indet. & 0.01 & 0.06 & 0.07 & & & & & & 0.07 \\
\hline \multicolumn{10}{|l|}{ Echinodermata } \\
\hline \multicolumn{10}{|l|}{ Asteroidea } \\
\hline \multicolumn{10}{|l|}{ Brisingida } \\
\hline Brisingida msp. 1 ( 6 arms - orange $)$ & & 0.03 & 0.07 & & 0.08 & & & & \\
\hline Brisingida msp. 1 ( 8 arms - orange) & 0.02 & 0.08 & 0.04 & & & & & & 0.03 \\
\hline Brisingida msp. 3 ( 6 arms - white) & & 0.08 & 0.13 & 0.04 & 0.06 & 0.19 & 0.06 & & 0.07 \\
\hline Brisingida msp. 4 (9-10 arms) & 0.02 & 0.08 & & & & & & & \\
\hline Brisingida indet. & 0.04 & 0.02 & & 0.04 & & & & & \\
\hline \multicolumn{10}{|l|}{ Paxillosida } \\
\hline Solaster msp. & & 0.01 & & & & & & & \\
\hline Paxillosida cf. AST_009 and AST_007 & & 0.10 & 0.06 & & & & & & \\
\hline Paxillosida msp. 1 & 0.01 & & & 0.02 & & & & & \\
\hline Paxillosida msp. 2a & & 0.01 & & & & & & & \\
\hline Paxillosida msp. $2 b$ & & & 0.01 & & & & & & \\
\hline Paxillosida msp. 3 & & 0.02 & 0.02 & & & & & & \\
\hline Paxillosida msp. 4 & & 0.02 & & & & & & & \\
\hline Paxillosida msp. 1 & & & & & & & & 0.03 & \\
\hline Paxillosida indet. & & 0.13 & & & & & & & \\
\hline \multicolumn{10}{|l|}{ Velatida } \\
\hline \multicolumn{10}{|l|}{ Pterasteridae } \\
\hline Hymenaster msp. 2 & 0.01 & & & & & & & & \\
\hline Pteraster msp. & 0.03 & & & & & & & & \\
\hline Velatida cf. AST_014 & 0.02 & 0.04 & & & & & & & \\
\hline Velatida msp. 2 & & & & & & & 0.06 & & \\
\hline Velatida msp. 3 & & & & & & & & & 0.03 \\
\hline Asteroidea indet. & 0.06 & 0.09 & 0.16 & 0.02 & 0.06 & & & & \\
\hline Crinoidea & & & & & & & & & \\
\hline Comatulida & & & & & & & & & \\
\hline Bourgueticrinina msp. 1 & & & & & 0.09 & & & & 0.07 \\
\hline Comatulida msp. 1 & 0.26 & 0.31 & & & & & 0.06 & & \\
\hline Comatulida msp. 2 & & & & & & & & & 0.07 \\
\hline
\end{tabular}


Table 2. Continued.

\begin{tabular}{|c|c|c|c|c|c|c|c|c|c|}
\hline & \multicolumn{4}{|c|}{ Seamounts } & \multicolumn{5}{|c|}{ Nodule fields } \\
\hline & ROV2 & ROV4 & ROV9 & ROV15 & ROV3 & ROV8 & ROV10 & ROV13 & ROV14 \\
\hline & ind. $100 \mathrm{~m}^{-2}$ & ind. $100 \mathrm{~m}^{-2}$ & ind. $100 \mathrm{~m}^{-2}$ & ind. $100 \mathrm{~m}^{-2}$ & ind. $100 \mathrm{~m}^{-2}$ & ind. $100 \mathrm{~m}^{-2}$ & ind. $100 \mathrm{~m}^{-2}$ & ind. $100 \mathrm{~m}^{-2}$ & ind. $100 \mathrm{~m}^{-2}$ \\
\hline \multicolumn{10}{|l|}{ Hyocrinida } \\
\hline Hyocrinidae small msp. & & & & & 0.11 & 0.12 & & & \\
\hline Hyocrinidae msp. 1 & & 0.04 & 0.01 & & & & & & \\
\hline Crinoidea red msp. & 0.03 & 0.33 & & 0.08 & & & & & \\
\hline Crinoidea golden msp. & 0.02 & 0.08 & & & & & & & \\
\hline Crinoidea msp. 1 & & & & & & & & & 0.03 \\
\hline Crinoidea indet. & 0.01 & 0.09 & 0.01 & 0.02 & & 0.06 & & & \\
\hline \multicolumn{10}{|l|}{ Echinoidea } \\
\hline \multicolumn{10}{|l|}{ Aspidodiadematidae } \\
\hline Aspidodiadematidae msp. 1 & & & & & 1.19 & 1.19 & 0.71 & & \\
\hline Aspidodiadematidae msp. 2 & & 0.04 & & & & 0.06 & & & \\
\hline Aspidodiadematidae soft msp. & & & & 0.02 & & & & & \\
\hline Aspidodiadematidae spiny msp. & 0.02 & & & 0.14 & & & & & \\
\hline \multicolumn{10}{|l|}{ Urechinidae } \\
\hline Urechinidae msp. 1_Nodules & & & & & & & & & 0.03 \\
\hline Urechinidae msp. 3 & 0.03 & 0.01 & 0.13 & & & & & & \\
\hline Urechinidae msp. 2_Nodules & & & & & & & & & 0.03 \\
\hline Urechinidae msp. 3_Nodules & & & & & 0.02 & & & & \\
\hline Urechinidae msp. 4_Nodules & & & & & & & & 0.03 & 0.07 \\
\hline Urechinidae msp. 1 & 0.03 & 0.15 & 0.04 & & & & & & \\
\hline Urechinidae msp. 2 & 0.03 & 0.01 & & & & & & & \\
\hline Urechinidae msp. 4 & 0.06 & 0.28 & 0.06 & & & & & & \\
\hline Urechinidae msp. 5 & 0.01 & & & & & & & & \\
\hline Urechinidae msp. 6 & 0.01 & & & & & & & & \\
\hline Urechinidae msp. 7 & 0.01 & & & & & & & & \\
\hline Urechinidae indet. & 0.02 & 0.02 & 0.01 & & & & & & \\
\hline Echinoidea indet. & 0.01 & & & & & & & & \\
\hline \multicolumn{10}{|l|}{ Holothuroidea } \\
\hline \multicolumn{10}{|l|}{ Elasipodida } \\
\hline \multicolumn{10}{|l|}{ Elpidiidae } \\
\hline Elpidiidae double velum msp. & & & & & & & 0.06 & & \\
\hline Elpidiidae msp. 1 & & & & & & & & 0.03 & 0.03 \\
\hline Amperima msp. & 0.02 & & & & & & & & \\
\hline Amperima msp. 1 & & & & & 0.02 & & & & \\
\hline Peniagone cf. palmata msp. & & & & & & 0.06 & 0.12 & & \\
\hline Peniagone tulip msp. & & & & & & & 0.06 & & \\
\hline Peniagone cf. leander & & & & & & 0.06 & 0.06 & & \\
\hline Peniagone msp. & 0.02 & 0.02 & & & & & & & \\
\hline Peniagone purple msp. & & & & & & & & & 0.03 \\
\hline Peniagone white or transparent msp. & & & & & 0.02 & & & 0.03 & 0.03 \\
\hline Peniagone indet. & & & & & 0.04 & & & & \\
\hline \multicolumn{10}{|l|}{ Laetmogonidae } \\
\hline Laetmogonidae msp. 1 & 0.04 & 0.09 & & & & & & & \\
\hline Laetmogonidae msp. 2 & 0.03 & & & & & & & & \\
\hline Laetmogonidae msp. 3 & & & & & & & 0.06 & & 0.03 \\
\hline \multicolumn{10}{|l|}{ Pelagothuriidae } \\
\hline Enypniastes sp. & & & & & & & & & 0.03 \\
\hline \multicolumn{10}{|l|}{ Psychropotidae } \\
\hline Benthodytes cf. incertae purple msp. & & 0.03 & 0.01 & & & & & & \\
\hline Benthodytes cf. incertae red msp. & & 0.09 & & & & & & & \\
\hline Benthodytes msp. & & 0.04 & & & & & & & \\
\hline Benthodytes msp. 1 & & & & & & & & & 0.03 \\
\hline Benthodytes pink msp. & & & & 0.02 & & & & & \\
\hline Benthodytes purple msp. & & & 0.01 & & & & & & \\
\hline Benthodytes red msp. & & 0.01 & 0.01 & & & & & & \\
\hline Psychropotes cf. semperiana & & & & & & & & 0.03 & \\
\hline
\end{tabular}


Table 2. Continued.

\begin{tabular}{|c|c|c|c|c|c|c|c|c|c|}
\hline & \multicolumn{4}{|c|}{ Seamounts } & \multicolumn{5}{|c|}{ Nodule fields } \\
\hline & ROV2 & ROV4 & ROV9 & ROV15 & ROV3 & ROV8 & ROV10 & ROV13 & ROV14 \\
\hline & ind. $100 \mathrm{~m}^{-2}$ & ind. $100 \mathrm{~m}^{-2}$ & ind. $100 \mathrm{~m}^{-2}$ & ind. $100 \mathrm{~m}^{-2}$ & ind. $100 \mathrm{~m}^{-2}$ & ind. $100 \mathrm{~m}^{-2}$ & ind. $100 \mathrm{~m}^{-2}$ & ind. $100 \mathrm{~m}^{-2}$ & ind. $100 \mathrm{~m}^{-2}$ \\
\hline Psychropotes longicauda & & & & & & & 0.12 & & \\
\hline Psychropotes msp. 3 & & & & & 0.02 & & 0.06 & & \\
\hline Psychropotes verrucosa & & & & & 0.08 & 0.06 & & & \\
\hline Psychropotidae msp. 1_Nodules & & & & & 0.02 & 0.06 & 0.06 & & \\
\hline Psychropotidae msp. 1 & & 0.07 & 0.01 & & & & & & \\
\hline Psychropotidae msp. 2_Nodules & & & & & & 0.19 & & & \\
\hline Psychropotidae msp. 2 & & 0.01 & & & & & & & \\
\hline Psychropotidae msp. 3 & & & & & 0.04 & 0.06 & & & \\
\hline Psychropotidae msp. 4 & & & & & & 0.06 & & & \\
\hline Psychropotidae red msp. & 0.02 & & & & & & & & \\
\hline Psychropotidae indet. & 0.16 & 0.09 & & 0.02 & & & & & \\
\hline \multicolumn{10}{|l|}{ Holothuriida } \\
\hline \multicolumn{10}{|l|}{ Mesothuriidae } \\
\hline Mesothuria msp. & 0.01 & 0.02 & & & & & & & \\
\hline \multicolumn{10}{|l|}{ Synallactida } \\
\hline \multicolumn{10}{|l|}{ Deimatidae } \\
\hline Deima msp. & & 0.01 & & & & & & & \\
\hline Deimatidae - irregular papillae length - msp. & & 0.05 & 0.01 & & & & & & \\
\hline Oneirophanta msp. & 0.01 & & 0.02 & & & & & & \\
\hline Deimatidae indet. & & 0.01 & 0.01 & & & & & & \\
\hline \multicolumn{10}{|l|}{ Synallactidae } \\
\hline Benthothuria msp. & & & & 0.08 & & & & & \\
\hline Paelopatides orange msp. & 0.01 & 0.01 & & & & & & & \\
\hline Synallactes msp. 1 (Synallactidae purple msp.) & 0.01 & & & & & & & & \\
\hline Synallactes msp. 2 & & 0.01 & & & & & & & \\
\hline Synallactes msp. 2 pink & & & & & 0.04 & 0.25 & 0.06 & & \\
\hline Synallactes msp. 2 pink (smooth) & 0.03 & 0.02 & & & & 0.31 & & & \\
\hline Synallactes sandy-coloured msp. & 0.02 & & & & & & & & \\
\hline Synallactes white msp. & 0.02 & & & & 0.70 & 0.19 & 0.30 & & 0.03 \\
\hline Synallactidae indet. & 0.04 & & & & & & & & \\
\hline \multicolumn{10}{|l|}{ Persiculida } \\
\hline \multicolumn{10}{|l|}{ Molpadiodemidae } \\
\hline Molpadiodemas msp. & & 0.02 & & & & & & & \\
\hline \multicolumn{10}{|l|}{ Pseudostichopodidae } \\
\hline Pseudostichopus msp. & & & & & & 0.06 & & & \\
\hline Molpadiodemas and Mesothuria & & & & & 0.06 & 0.12 & 0.06 & & 0.07 \\
\hline Holothuroidea indet. & 0.17 & 0.15 & 0.04 & 0.04 & 0.06 & 0.06 & 0.12 & & \\
\hline \multicolumn{10}{|l|}{ Ophiuroidea } \\
\hline Ophiuroidea msp. 1 & & & & & 0.02 & 0.06 & 0.06 & & \\
\hline Ophiuroidea msp. 3 & & & & & & 0.12 & & & \\
\hline Ophiuroidea msp. 5 & 0.02 & 0.39 & 0.49 & & & & & 0.03 & 0.10 \\
\hline Ophiuroidea msp. 6 & & 0.03 & 0.01 & & 0.32 & 1.31 & 0.65 & 0.17 & 0.44 \\
\hline Ophiuroidea msp. 4 & 0.04 & 0.21 & & & 0.11 & & & & \\
\hline Ophiuroidea msp. 7 & & 0.01 & & & & & & & \\
\hline Ophiuroidea indet. & & 0.02 & 0.04 & 0.08 & 5.67 & 6.68 & 7.31 & & 0.13 \\
\hline \multicolumn{10}{|l|}{ Enteropneusta } \\
\hline Enteropneusta msp. 1 cf. Yoda & & 0.10 & & & & & & & \\
\hline Enteropneusta msp. 2 cf. Saxipendium msp. & 0.07 & & & & & & & & \\
\hline \multicolumn{10}{|l|}{ Mollusca } \\
\hline \multicolumn{10}{|l|}{ Gastropoda } \\
\hline Limpet & & & 0.01 & & & & & & \\
\hline Gastropoda msp. 1 & & & 0.02 & & & & & & \\
\hline Polyplacophora & 0.04 & & & 0.04 & & & & & 0.07 \\
\hline Gastropoda indet. & & & & & & 0.06 & & & \\
\hline Cephalopoda & & & & & & & & & \\
\hline Octopoda msp. 1 & 0.01 & & & & & & & & \\
\hline Pisces* & 0.33 & 0.28 & 0.07 & 0.06 & 0.47 & 0.19 & 0.48 & 0.17 & 0.17 \\
\hline
\end{tabular}


Table 2. Continued.

\begin{tabular}{|c|c|c|c|c|c|c|c|c|c|}
\hline & \multicolumn{4}{|c|}{ Seamounts } & \multicolumn{5}{|c|}{ Nodule fields } \\
\hline & ROV2 & ROV4 & ROV9 & ROV15 & ROV3 & ROV8 & ROV10 & ROV13 & ROV14 \\
\hline & ind. $100 \mathrm{~m}^{-2}$ & ind. $100 \mathrm{~m}^{-2}$ & ind. $100 \mathrm{~m}^{-2}$ & ind. $100 \mathrm{~m}^{-2}$ & ind. $100 \mathrm{~m}^{-2}$ & ind. $100 \mathrm{~m}^{-2}$ & ind. $100 \mathrm{~m}^{-2}$ & ind. $100 \mathrm{~m}^{-2}$ & ind. $100 \mathrm{~m}^{-2}$ \\
\hline \multicolumn{10}{|l|}{ Porifera } \\
\hline \multirow{2}{*}{\multicolumn{10}{|c|}{ Demospongiae }} \\
\hline & & & & & & & & & \\
\hline Cladorhizidae msp. 1 & & & 0.02 & & & & & & 0.07 \\
\hline Cladorhizidae msp. 1 (soft) & & & & & & & & & 0.03 \\
\hline Cladorhizidae msp. 2 & & & & & & & & 0.03 & 0.03 \\
\hline Cladorhizidae msp. 3 & & & & & & & & & 0.03 \\
\hline Cladorhizidae msp. 4 & & & & & 0.02 & & & 0.06 & 0.13 \\
\hline Cladorhizidae msp. 5 & & & & & & 0.06 & & & \\
\hline Cladorhizidae msp. 6 & & & & & & 0.06 & & & \\
\hline Cladorhizidae indet. & & & & & & & & 0.03 & 0.07 \\
\hline \multirow{2}{*}{\multicolumn{10}{|c|}{ Hexactinellida }} \\
\hline Euplectellidae & & & & & & & & & \\
\hline Bathydorus spinosus & 0.01 & & & & & & & & \\
\hline Bolosoma sp. & & & & 0.02 & & & & & \\
\hline Corbitella discasterosa & 0.01 & & & & & & & 0.06 & \\
\hline Docosaccus maculatus & & & & & & 0.06 & & 0.03 & \\
\hline Docosaccus nidulus & & & & & & 0.06 & & & \\
\hline Holascus spp. & & & & & 0.19 & 0.12 & 0.06 & 0.03 & 0.03 \\
\hline Hyalostylus schulzei & & & & & & & & 0.03 & \\
\hline Hyalostylus sp. & & 0.02 & 0.15 & 0.02 & & & & 0.03 & 0.03 \\
\hline Saccocalyx pedunculatus & & & & 0.06 & & & & & \\
\hline Saccocalyx sp. & 0.04 & 0.02 & 0.02 & & & & & & \\
\hline \multicolumn{10}{|l|}{ Euretidae } \\
\hline Bathyxiphus subtilis & & & & & & & & 0.03 & \\
\hline Chonelasma bispinula & & & & & & & 0.06 & & \\
\hline Chonelasma choanoides & 0.01 & & & & & & & & \\
\hline Chonelasma sp. & & & 0.01 & & & & & & \\
\hline \multicolumn{10}{|l|}{ Hyalonematidae } \\
\hline Hyalonema spp. & & 0.02 & 0.10 & 0.02 & 0.11 & 0.31 & 0.24 & 0.08 & 0.23 \\
\hline \multicolumn{10}{|l|}{ Rossellidae } \\
\hline Caulophacus sp. & & 0.06 & 0.07 & 0.02 & 0.17 & 0.06 & 0.06 & & \\
\hline Crateromorpha sp. & & 0.02 & & 0.02 & & & & & \\
\hline Rossellidae gen. sp. & 0.04 & 0.01 & 0.02 & & & & & & \\
\hline \multicolumn{10}{|l|}{ Pheronematidae } \\
\hline Poliopogon sp. & & & & 0.02 & & & & & \\
\hline Hexactinellida - foliose sponge msp. & 0.01 & 0.02 & 0.01 & 0.06 & & & & & \\
\hline Hexactinellida - stalked & & & & & 0.26 & 0.50 & 0.53 & & \\
\hline Hexactinellida black msp. & & 0.01 & & & & & & & \\
\hline Hexactinellida indet. & 0.20 & 0.20 & 0.52 & 0.12 & 0.98 & 1.06 & 1.60 & 0.45 & 0.37 \\
\hline Pycnogonida & 0.02 & & 0.01 & & & & & & 0.03 \\
\hline \multicolumn{10}{|l|}{ Tunicata } \\
\hline \multicolumn{10}{|l|}{ Octacnemidae } \\
\hline Megalodicopia msp. 1 & 0.02 & 0.01 & 0.01 & & & & & & \\
\hline Megalodicopia msp. 2 & & & & & & & & & 0.03 \\
\hline Dicopia msp. & 0.04 & & & & & & & & \\
\hline \multicolumn{10}{|l|}{ Pyuridae } \\
\hline Culeolus msp. & & & & & & & & & 0.03 \\
\hline Tunicata indet. & 0.02 & 0.01 & 0.01 & 0.02 & & & & & \\
\hline Paleodictyon nodosum & & & & & & & & 0.03 & \\
\hline
\end{tabular}

* Taxa left out of the statistical analyses due to lack of representativity. 
(a)

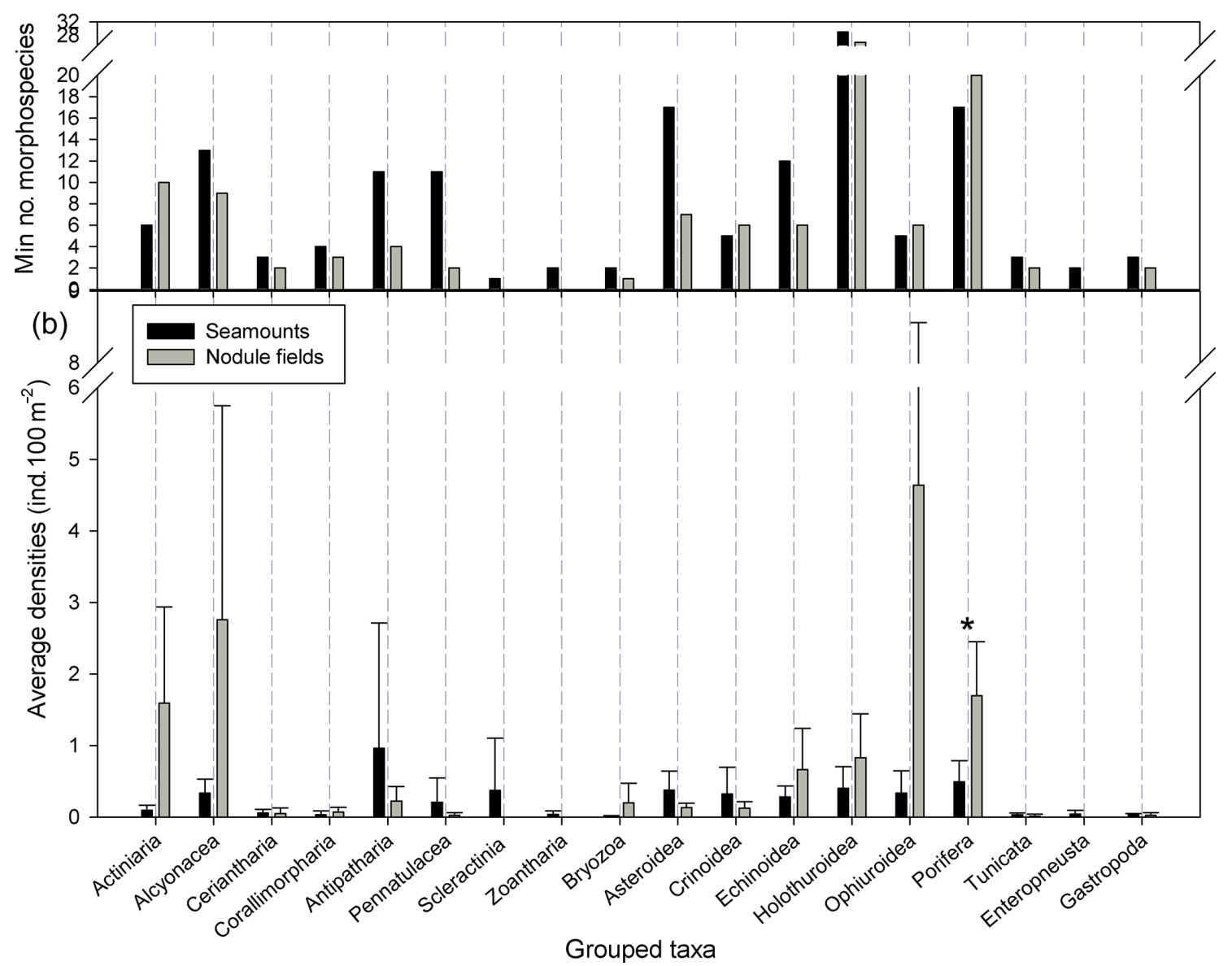

Figure 3. (a) Minimum number of morphospecies per taxon and ecosystem and (b) average densities at higher taxa levels per ecosystem and standard deviation. ${ }^{*}$ Significant difference in density $(t=-3.7, p<0.05)$.

and lastly Rüppel (0.31 ind. $100 \mathrm{~m}^{-2}$; Table 2, Fig. A1c). Six Porifera families were annotated featuring $>7$ to $>10$ morphospecies per transect (Table 2). Cladorhizidae (two individuals) were only observed along the Heip transect, and one Poliopogon sp. (Pheronematidae) was observed along the Mann Borgese transect. Rossellidae gen. sp. nov. was present along three seamount transects, the exception being Mann Borgese.

Overall Echinodermata densities were highest at Senckenberg seamount ( 3.5 ind. $100 \mathrm{~m}^{-2}$ ), adding up to $51 \%$ of all image annotations for this transect. For comparison, echinoderms at Heip (1.5 ind. $\left.100 \mathrm{~m}^{-2}\right)$ and Rüppel $\left(1.4\right.$ ind $\left.100 \mathrm{~m}^{-2}\right)$ were responsible for $37 \%$ and $32 \%$ of all image annotations for these transects, followed by Mann Borgese $\left(0.62\right.$ ind. $\left.100 \mathrm{~m}^{-2}\right)$ with $8.2 \%$ of the annotations. The number of morphospecies for all echinoderm taxa (Asteroidea, Echinoidea, Holothuroidea and Crinoidea) was also highest on these two seamounts in the BGR area (Fig. A1, Table 2). Crinoidea and Holothuroidea densities were highest at Senckenberg ( 0.9 and 0.7 ind. $100 \mathrm{~m}^{-2}$ respectively). The holothuroid families of Elpidiidae and Laetmogonidae were only observed at Senckenberg and Rüppel (BGR). Psychropotidae and Synallactidae were observed on all seamounts, represented by different morphospecies. Deimatidae were not observed on Mann Borgese but were present along the three other seamount transects, again with different morphospecies and densities. Velatid Asteroidea were only observed at Senckenberg and Rüppel (BGR), while Brisingida and Paxillosida were observed on all four seamounts. Aspidodiadematid Echinoidea were absent from the Heip transect and urechinid Echinoidea were absent from the Mann Borgese transect.

A species accumulation curve (Fig. 4a) confirmed the limitations of the restricted and exploratory nature of the sampling as no asymptote was reached. The rarefaction curves (Fig. 4b) showed that the transects with the most faunal observations, which corresponded here to the longer transects, were more diverse. However, at smaller sample sizes, curves did not cross, thus maintaining the differences observed at larger sample sizes with the Senckenberg transect (ROV04) 

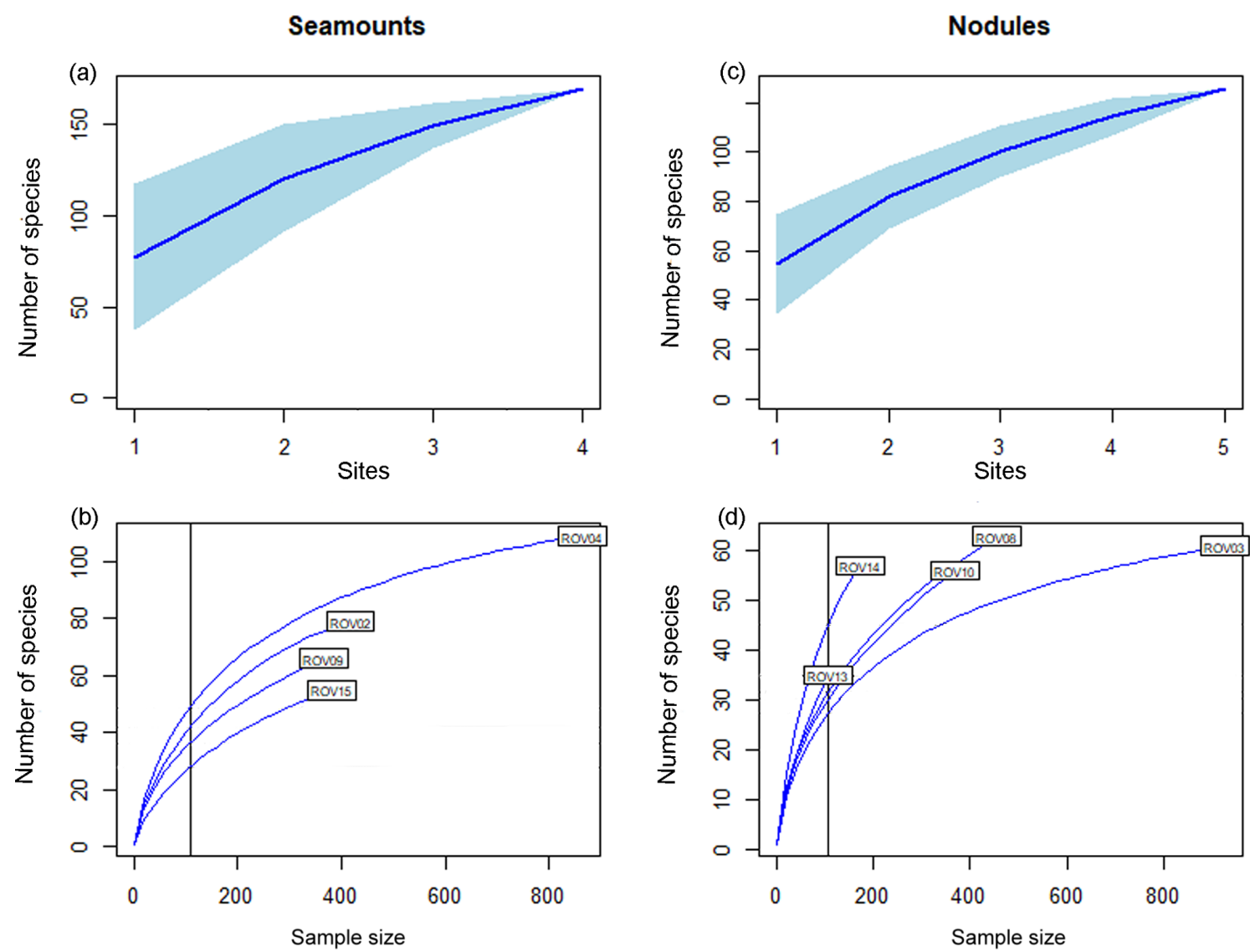

Figure 4. Species accumulation $(\mathbf{a}, \mathbf{c})$ and rarefaction curves $(\mathbf{b}, \mathbf{d})$ for the seamount $(n=4)$ and nodule field $(n=5)$ transects. Seamount dives: ROV02 is Rüppel (BGR); ROV04 is Senckenberg (BGR); ROV09 is Heip (GSR); ROV15 is Mann Borgese (APEI3) in (b). Nodule field dives: ROV03 was carried out in the BGR area, ROV08 and ROV10 in the GSR area, and ROV13 and ROV14 in APEI3, presented in (d). Sample size is the number of individuals. Vertical line in the lower panel shows sample size $=100$.

as most diverse followed by Rüppel (ROV02; both transects BGR). The video transect carried out at Mann Borgese (ROV15, APEI3) was the least diverse.

A comparison of all morphospecies observed along the four transects was presented in a Venn diagram (Fig. 5a). Each seamount transect was characterised by its highest number of unique morphospecies, only observed along the transect in question and not elsewhere. Only three morphospecies were present along all seamount transects, namely Ceriantharia msp. 2, a small red galatheid crab and a foliose sponge. The highest number of overlapping morphospecies ( $n=16)$ was observed between Rüppel and Senckenberg, both in the BGR area (Fig. 5a). Mann Borgese showed the smallest degree of overlap with the other transects (Fig. 5a).

About $57 \%$ of all sessile fauna was associated with predominantly hard substrata, followed by $31 \%$ on the mixed substrata. For the mobile taxa, the pattern was less pronounced with $41 \%$ and $42 \%$ associated with predominantly hard and mixed hard and soft substrata respectively. The amount of predominantly hard and soft substrata was negatively correlated, though not significantly. This was due to the equal amounts (40\%-60\%) of mixed hard and soft substrata. Over all seamount transects pooled together, no taxa were significantly correlated with the amount of hard substrata or with soft substrata. When looking at the individual transects, no significant correlations were found between taxa and substrata for ROV02, ROV04 or ROV09, most likely due to the equal distribution of the amount of hard, soft and mixed substrata. From this perspective, ROV15 stood out, as it was dominated by predominantly hard substrata $(56 \%)$. For this transect, Pennatulacea were significantly negatively correlated with the amount of hard substrata and Zoantharia and Octocorallia were significantly and positively correlated with hard substrata, as were Ophiuroidea, Asteroidea, Crinoidea and Mollusca.

Due to the limited sample size, the representativity of the observed biological patterns remains to be corroborated by a more elaborate sampling strategy. 

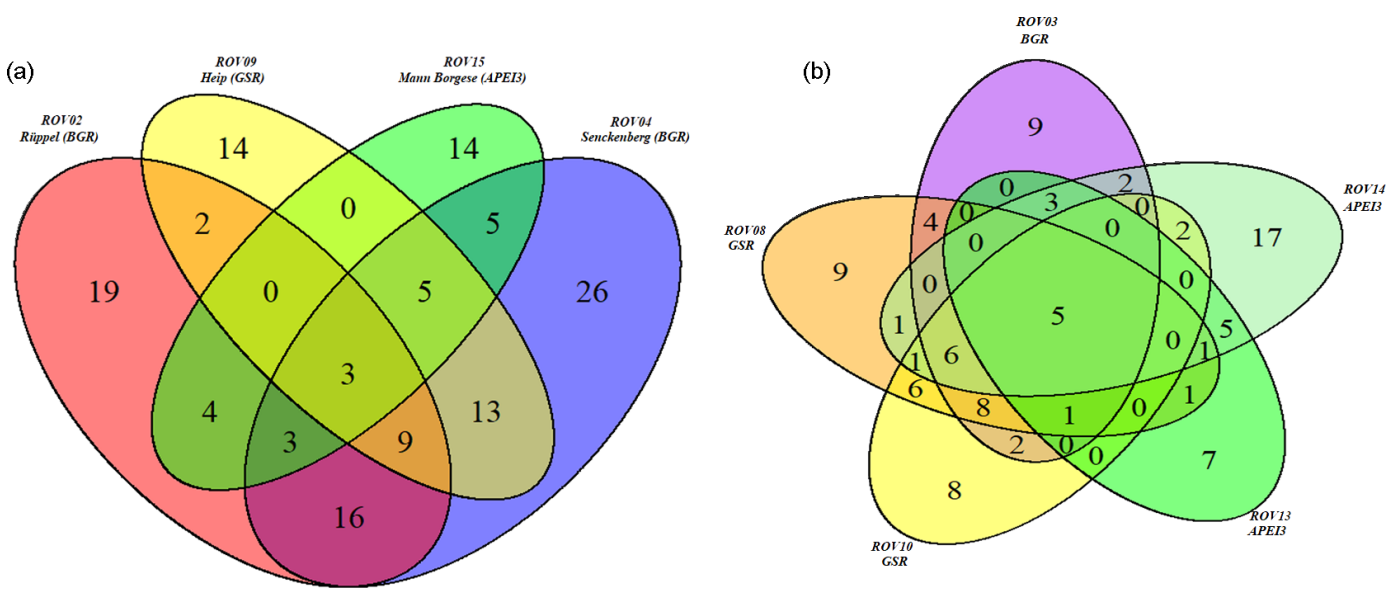

Figure 5. A Venn diagram showing the unique and shared morphospecies among seamount video transects. Values are indicative rather than absolute due to different transect lengths and differences in richness. Panel (a) features seamount transects and panel (b) features the five nodule field transects. Colour codes are adopted in panels, with APEI3 nodule transects in green, related to the Mann Borgese seamount transect. The BGR (ROV03) transect is purple, corresponding to BGR seamount transects (red is Rüppel, and blue is Senckenberg). GSR transects (ROV08 and ROV09) are shades of yellow.

\subsection{Comparison of seamount and nodule field faunal composition and variation}

The faunal composition and richness of the nodule transects can be consulted in Figs. 3, A1 and Table 2. The only taxon showing significant difference in density between seamounts and nodule fields was the Porifera $(t$ test assuming unequal variances, $t=-3.7, p<0.05)$. In concordance with the seamount transect, the species accumulation curve of the nodule transects did not reach an asymptote either (Fig. 4c). The rarefaction curves showed that the relations among transects were less straightforward for the nodule transects versus the seamount ones and crossed at smaller sample sizes ( $<100$ individuals; Fig. 4d). ROV13 and ROV14 transects (both APEI3) were the longest in distance travelled (Table 1) but featured fewer faunal observations. At small sample sizes, the richness along ROV13 and ROV14 was highest. ROV08 and ROV10 (both GSR) showed parallel curves with ROV08 being more diverse (Fig. 4d).

A Venn diagram showing the morphospecies overlap among the nodule transects showed a total of five species reoccurring along all five transects (Fig. 5b). These were Munnopsidae msp. 1 (Isopoda, Crustacea), Actiniaria msp. 7 (Cnidaria), Ophiuroidea msp. 6 (Echinodermata), Holascus sp. and Hyalonema sp. (Hexactinellida, Porifera). There was a high number of unique morphospecies for each transect, though not as high as for the seamount transects (Fig. 5). ROV13 and ROV14 (both APEI3) showed the least overlap with the other transects, which is similar to what was observed on the seamounts.

Observations and quantifications of morphospecies confirmed the high degree of dissimilarity between the two neighbouring ecosystems. Porifera, Ophiuroidea (Echino- dermata), Actiniaria and Alcyonacea (Cnidaria) were more abundant in nodule fields (Fig. 3). These taxonomic groups were also most diverse in nodule fields (i.e. with the highest number of morphospecies), the exception being the Alcyonacea which featured more morphospecies on the seamounts (12 and 8 morphospecies for seamounts and nodule fields respectively; Fig. 3). Of all Porifera, Cladorhizidae were more diverse in nodule fields than on seamounts ( 7 and 1 morphospecies respectively).

There were only 21 morphospecies $(10 \%)$ that were observed both on seamounts and in nodule fields (Fig. 6). While this subset of morphospecies occurred in both ecosystems, it did so in very different densities, i.e. very abundant in one ecosystem and very low in abundance in the other - examples are Galatheidae small red msp. (Decapoda, Crustacea), Synallactes white msp. (Holothuroidea), Ophiuroidea msp. 5 and 6, Comatulida msp. 1 (Crinoidea), Hyalonema sp. and Hyalostylus sp. (both Hexactinellida, Porifera; Fig. 6).

Three Ophiuroidea morphospecies were present both on seamounts and in nodule fields (Figs. 2, 3 and 6). Most of the Ophiuroidea observed on the CCZ seamounts, that could be identified to a morphospecies level, were small and situated on hard substrata (morphospecies 5), while those in nodule fields (including morphospecies 6) were observed on the soft sediments. Morphospecies 6 was only rarely observed on the seamounts (Fig. A1b). Another easily recognisable morphospecies was found on Porifera, coral and animal stalks and was more abundant on seamounts than in nodule fields (morphospecies 4; Fig. A1b).

Crinoidea, Asteroidea (both Echinodermata) and Antipatharia (Cnidaria) were more abundant on the seamounts (Fig. 3). This coincided with a higher diversity of Asteroidea and Antipatharia on the seamounts as well. Crinoidea diver- 
Morphospecies overlap

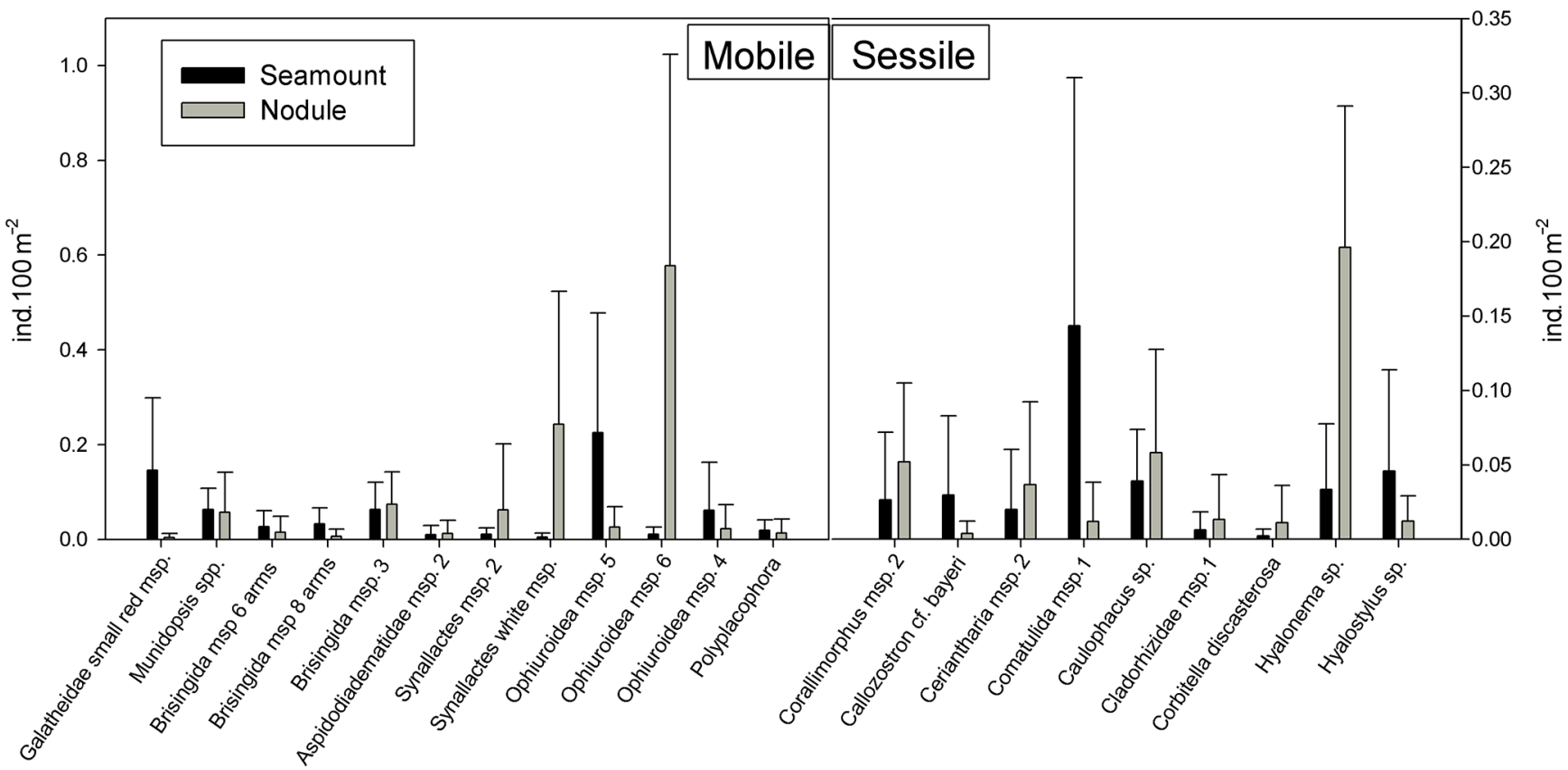

Figure 6. Morphospecies present along both seamount and nodule field transects and their average density (ind. $100 \mathrm{~m}^{-2}$ ) and standard deviation per ecosystem.

sity was similar (five and four morphospecies for seamounts and nodule fields respectively). Holothuroidea occurred in similar densities in both ecosystems (Fig. 3), though they were characterised by different morphospecies (Table 2, Fig. A1b). Overall densities of Echinoidea were comparable between seamounts and nodule fields, though for the nodule fields this was mostly due to one very abundant morphospecies, namely Aspidodiadematidae msp. 1, which was absent from the seamounts (Table 2, Fig. A1b). Besides this, Echinoidea were more diverse on seamounts (11 morphospecies vs. 5 in nodule fields).

There was no morphospecies overlap for Tunicata, Antipatharia and Actiniaria. Alcyonacea, Ceriantharia, Corallimorphidae and Crinoidea only shared one morphospecies between seamounts and nodule fields, namely Callozostron cf. bayeri, Ceriantharia msp. 2, Corallimorphus msp. 2 and Comatulida msp. 1 respectively (Fig. 6).

There were no observations of Enteropneusta, Scleractinia and Zoantharia (Cnidaria), Aphroditidae (Polychaeta), or holothuroid Deimatidae along the nodule fields transects (Table 2, Fig. A1). While Actinopterygii were left out of the analysis, it should be noted that fish observations were more diverse on the seamounts than in the nodule fields.

There was quite some faunal variation observed among the video transects of both seamounts and nodule fields (see Figs. 5 and 7). The (dis)similarities were analysed by a nMDS analysis, which grouped the nine different video transects based on their taxonomic composition. Despite the large intra-ecosystem variation, they could be pooled into two distinct groups separating the nodule fields from the seamounts (Fig. 7a). Within each group, BSR and GSR transects were more similar to one another both for seamounts and nodule fields, whilst APEI3 transects stood out more.

The Kendall's coefficient of concordance ( $W$; Legendre, 2005) corroborated the existence of two significantly different species associations, whose composition corresponded to the fauna characterising the nodule fields $(W=2.03$, $p<0.001$, after 999 permutations) and the seamounts ( $W=$ 3.04, $p<0.001$, after 999 permutations).

Depth was fitted as a vector on top of the nMDS plot (Fig. 7b) and showed that the discrepancy in faunal composition between the two ecosystems also corresponded to a difference in depth, with the nodule transects all being situated below the $4000 \mathrm{~m}$ isobath and the seamount transects ranging from 1650 to $>3500 \mathrm{~m}$ (Fig. $7 \mathrm{~b}$ ).

\section{Discussion}

\subsection{Intra-ecosystem faunal variation}

Community composition varied markedly on seamounts and in nodule fields. The limited sampling ( $n=9$ transects), at different locations and additionally, for the seamounts, different depth ranges, precluded any general conclusions on quantifications of biodiversity per se. However, taking this into account, it was also the first time seamounts were visited in the area, thus giving first insights into the fauna inhabiting 

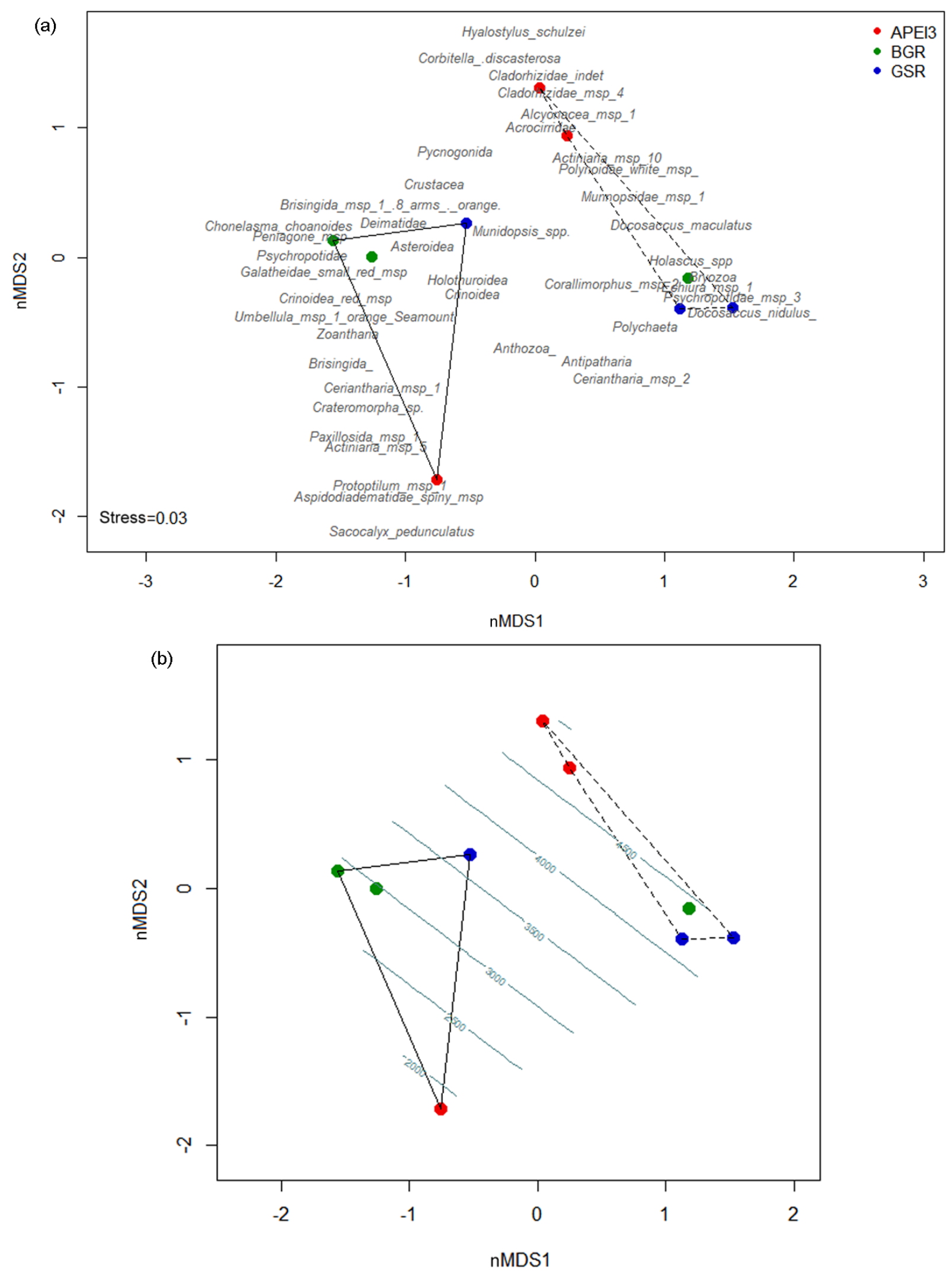

Figure 7. nMDS plot with faunal densities and Bray-Curtis distances. (a) presents the grouping of the video transects based on their faunal composition and (b) features the same plot but with depth as a vector fitting. Dotted lines group the nodule transects while the full line groups the seamount transects. 
these seamounts and allowing a first comparison with nodule faunal composition.

The two BGR seamount transects were most similar in faunal composition, followed by the Heip seamount transect (GSR). These seamount video transects were characterised by more similar depth ranges, and the two BGR transects were also geographically closest to each other. Although for seamounts, distance separating them might be a less determining factor than depth since (mega)faunal communities can be very different even between adjacent seamounts (Schlacher et al., 2014; Boschen et al., 2015). Overall, parameters that vary with depth, such as temperature, oxygen concentration, substratum type, food availability and pressure, are considered major drivers of species composition on seamounts (Clark et al., 2010; McClain et al., 2010). The quantification of the amount of hard and soft substrata was not distinctive enough to explain differences observed here. The difference in depth could also explain the higher dissimilarity with Mann Borgese (APEI3), which featured the shallowest transect and summit and was dominated by Antipatharia. Antipatharians were previously reported to be more dominant towards peaks compared to on mid-slopes at corresponding depths (Genin et al., 1986). Based on their filter-feeding strategy, Porifera (except carnivorous Cladorhizidae), were also thought to benefit from elevated topography (peaks) or exposed substrata analogous to corals (Genin et al., 1986; Clark et al., 2010), though no such pattern was apparent here. Porifera are notoriously difficult to identify based on imagery. Although the sampled individuals allowed for some identifications at the genus or species level (Kersken et al., 2018a, b), identifications remained hard to extrapolate across the different video transects. Generally, as in our study, seamount summits have been more intensively sampled (Stocks, 2009) although the little work that has been done at seamount bases and on deep slopes has indicated that these areas support distinct assemblages (Baco, 2007).

Among the nodule transects a considerable amount of variation in faunal composition was observed (this study; Vanreusel et al., 2016). The two APEI3 nodule transects (ROV13 and ROV14) stood out in terms of faunal composition, diversity and a low number of faunal observations. They were also the only two transects situated below the $4500 \mathrm{~m}$ isobaths. But rather than depth, the nodule coverage may be considered an important driving factor, since the density of nodule megafauna was shown to vary with nodule size and density or coverage (Stoyanova, 2012; Vanreusel et al., 2016; SimonLlédo et al., 2019). Here as well, the APEI3 transects were characterised by a high nodule coverage ( $\sim 40 \%-88 \%$; Vanreusel et al., 2016), whereas the BGR and GSR nodule transects (ROV3 for BGR and ROV8 and ROV10 for GSR) had a nodule coverage of $<30 \%$ and were also more similar in faunal composition (Vanreusel et al., 2016). Another factor that could be at play is the more oligotrophic surface waters of the northern CCZ which could be the cause of the overall lower faunal densities in APEI3 nodule fields (Vanreusel et al., 2016). Volz et al. (2018) corroborated this, with the location of the APEI3 site in proximity to the carbon-starved North Pacific Gyre being characterised by a reduced particulate organic carbon (POC) flux quantified as being $22 \%$ and $46 \%$ lower than the GSR and BGR areas respectively.

The species accumulation curves showed that an asymptote was reached neither on seamounts nor in nodule fields. Consequently, longer transect lengths might be necessary to representatively quantify and assess megafauna density and diversity (Simon-Lledó et al., 2019). In addition, for a first in-depth description and assessment of seamount fauna composition, one video transect is insufficient to describe the diversity and shifts in faunal assemblages of the surveyed seamounts. Rather, a more extensive imaging strategy should be developed, with a minimum transect length exceeding $1000 \mathrm{~m}$ (Simon-Llédo et al., 2019) and replicate transects carried out on different faces of the seamount, on slopes with varying degrees of exposure to currents and on different substrate types. Wider depth ranges should be taken into account as well. Alternatively, across-slope transects, following depth contours, should be considered as these could provide observation replicates for a given depth. Despite its limitations, this study gives first insights into the seamount-inhabiting megafauna of the eastern CCZ and an important first comparison with nodule fauna.

\subsection{Faunal (dis)similarities between seamounts and nodule fields}

In other areas, seamounts have been shown to share fauna with surrounding habitats (Clark et al., 2010) and could thus potentially serve as source populations for neighbouring environments (McClain et al., 2009). While generally few species seemed restricted to seamounts only (Clark et al., 2010), morphospecies in this study were revealed to be quite different between seamounts and nodule fields with little overlap between both. Despite the high degree of variation observed among all the video transects, these could be grouped into two distinctly separate clusters, separating nodule from seamount transects. The few overlapping morphospecies that did occur did so in different densities in each ecosystem, implying different roles or importance in the ecological community and its functioning.

Overall, nodule fields showed higher faunal densities than seamounts. Shifts in density patterns between nodule fields and seamounts were more evident in a number of taxa, where the variety of morphospecies and feeding strategy within each group was likely to be at play. An example of this are the Echinodermata, which include Asteroidea (predators and filter feeders (Brisingida), Crinoidea (filter feeders), Echinoidea (deposit feeders), Holothuroidea (deposit feeders) and Ophiuroidea (omnivores). Asteroidea were more abundant on seamounts, and both Echinoidea and Asteroidea were more diverse in this ecosystem as well. Ophiuroidea were most abundant in the nodule fields (ratio 7 to 1 when com- 
pared to seamounts). The same ophiuroid morphospecies were present on seamounts and in nodule fields but in very different abundances, and they were observed on different substrata types, which indicates different lifestyles, feeding behaviour and corresponding dietary specialisations (Persons and Gage, 1984). Previously it has already been demonstrated that Ophiuroidea did not show high levels of richness or endemism on seamounts (O'Hara, 2007). In nodule fields, Ophiuroidea were often observed in association with xenophyophores (Amon et al., 2016; this study), and a similar observation was made on east Pacific seamounts off Mexico (Levin et al., 1986), though no such associations were observed on the seamounts studied here.

Holothuroidea densities were thought to possibly decrease when less soft sediment was available since they feed mainly on the upper layers of the soft bottom sediment (Bluhm and Gebruk, 1999). No significant link was established between holothuroid densities and the amount of hard substrata in this study, but their community composition varied distinctly between nodule fields and seamounts with more families being observed at the latter. Additionally, on the seamounts, many holothurians were observed on top of rocks, possibly reflecting different feeding strategies and explaining the observations of different morphospecies. Geographical variations, different bottom topography, differences in nodule coverages and sizes and/or an uneven distribution of holothurians on the sea floor are thought to play a role in holothuroid community composition (Bluhm and Gebruk, 1999). On the other hand, it has been proposed that variability in deep-sea holothuroid abundance depends primarily on depth and distance from continents (see Billet, 1991, for a review).

Stalked organisms, such as Crinoidea (Echinodermata) and Hexactinellida (except for Amphidiscophora, Porifera) rely on hard substrata for their attachment and are considered to be among the most vulnerable organisms where mining is concerned. Crinoidea were more abundant on seamounts, possibly because hard substrata were less limiting than in the nodule fields. Porifera densities (stalked and non-stalked) varied among all analysed transects, revealing no particular trends in abundance. However, the species composition of deep-sea glass sponge communities from seamounts and polymetallic nodule fields was distinctly different. Polymetallic nodule field communities were dominated by widely distributed genera such as Caulophacus and Hyalonema, whereas seamount communities seemed to have a rather unique composition represented by genera like Saccocalyx.

Corals were generally considered to be more abundant on seamounts than in adjacent areas, due to their ability to feed on a variety of planktonic or detritus sources suspended in the water column (Rowden et al., 2010). In this study, the Alcyonacea densities were lower along the seamounts than along the nodule transects. The majority of Alcyonacea morphospecies of the seamounts did not occur in the nodule fields and vice versa, with the exception of Callozostron cf. bayeri which was also present in the nodule fields but in very low densities (one-eighth of those observed on seamounts). The Antipatharia were most abundant on the Mann Borgese seamount (APEI3) compared to all other transects. The depth difference of more than $3000 \mathrm{~m}$ between this particular seamount and the nodule fields could explain the abundance in Antipatharia which were shown to be more abundant at lower depths (Genin et al., 1986). The additional presence of Pennatulacea on seamounts, a taxon that was virtually absent from the nodule field transects and that appeared to be more linked to predominant soft substrata on seamounts, resulted in completely distinct coral communities for each ecosystem.

Actiniaria were denominated the second most common group in CCZ nodule fields, after the xenophyophores (Kamenskaya et al., 2015), and, in our study, were also more abundant in nodule fields than on seamounts. Depending on the species and feeding strategy, the ratio of hard to soft substrata and the species' preference for either one could play a role. Since morphospecies were distinct between seamounts and nodule fields, their role in the respective communities are likely to differ as well. Combinations of deposit feeding and predatory behaviour in Actiniaria have been observed, as well as burrowing activity, a preference for attachment to hard substrata and exposure to currents (Durden et al., 2015a; Lampitt and Paterson, 1987; Riemann-Zürneck, 1998), all of which are factors that could influence the differences in morphospecies observed.

Some taxa were only observed on the seamounts in this study, while they occurred in nodule fields elsewhere, be it in low densities. For instance, Enteropneusta, which in this study were found only on seamounts, were observed previously in CCZ nodule fields though observations were rather rare (Tilot, 2006). They appeared more abundant in the nodule fields of the deep Peru Basin (DISCOL area), though a wide range in abundances was displayed there as well (Bluhm, 2001). The exception were the Scleractinia, which were quite common on seamounts, as also reported in other studies (e.g. Baco, 2007; Rowden et al., 2010), but distinctly absent from nodule fields.

Explanations for the discrepancies in faunal composition and the low degree of morphospecies overlap between seamount and nodule fields, as observed here, can be multiple. For one, nodules may not be considered a plain hard substratum, with their metal composition, microbial colonisation and the nodule-sediment interface influencing the epifaunal and associated megafaunal composition. The possibility of a specific deep-sea faunal community that tolerates or benefits from manganese substrata has previously been proposed (Mullineaux, 1988). The comparison between seamounts and nodule fields as two neighbouring hard-substrata ecosystems also entailed a comparison between depth gradients and possible thresholds ( $>4000 \mathrm{~m}$ for nodule fields and $1500>x<$ $4000 \mathrm{~m}$ for seamounts). Related to this is the steepness of the seamount slope and its current exposure playing a role in faunal colonisation (Genin et al., 1986; Rappaport et al., 1997). Other studies have shown that habitat heterogene- 
ity increased megafaunal diversity on seamounts (Raymore, 1982) and elsewhere, such as in abyssal plains (Lapointe and Bourget, 1999; Durden et al., 2015b; Leitner et al., 2017; Simon-Llédo et al., 2019). From this perspective the smallerscale substratum heterogeneity transcending the ratio of hard to soft substrata or amount of hard substrata could play a role as well.

\section{Conclusions}

Based on our current knowledge, seamounts appear inadequate as refuge areas to help maintain nodule biodiversity. In order to conclusively exclude seamount habitats as a refuge for nodule fauna, a more comprehensive sampling should be carried out. The sampling strategy used in this study lacked replicates and uniformity and was limited in sample size. Seamount bases should be taken into consideration as well, as they can be characterised by distinctly different assemblages than the summits and feature depth ranges more similar to nodule fields.

While their role as refuge areas for nodule field fauna is currently debatable, the possible uniqueness of the seamount habitat and its inhabiting fauna implies that seamounts need to be included in management plans for the conservation of the biodiversity and ecosystems of the CCZ. 


\section{Appendix A}

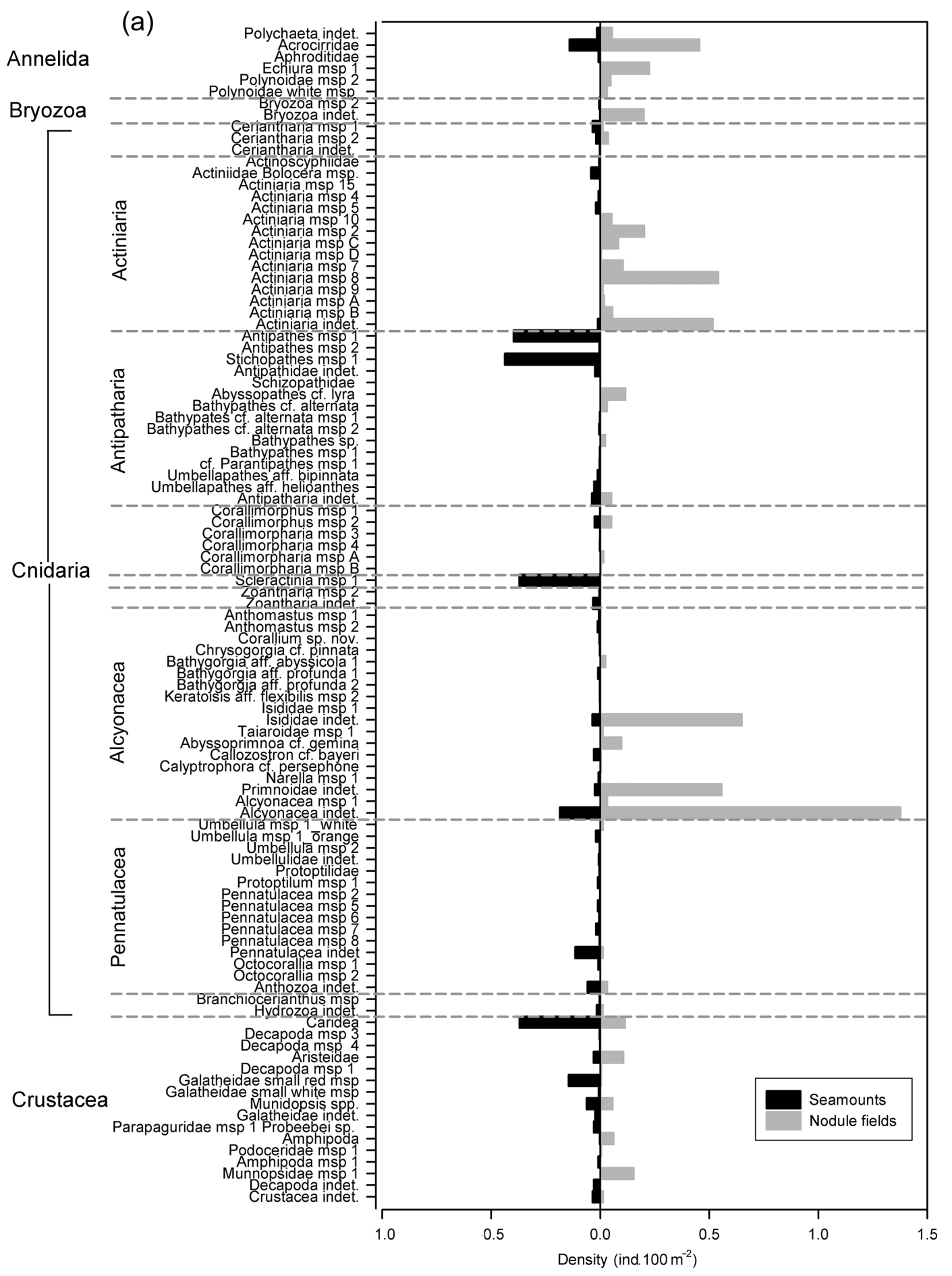

Figure A1. Back-to-back histogram comparing average densities of morphospecies and taxa (ind. $100 \mathrm{~m}^{-2}$ ) for seamount $(n=4)$ and nodule field $(n=5)$ video transects. (a) Annelida, Bryozoa, Cnidaria and Crustacea, (b) Echinodermata and (c) Mollusca, Porifera, Hemichordata and Chordata (Tunicata). 


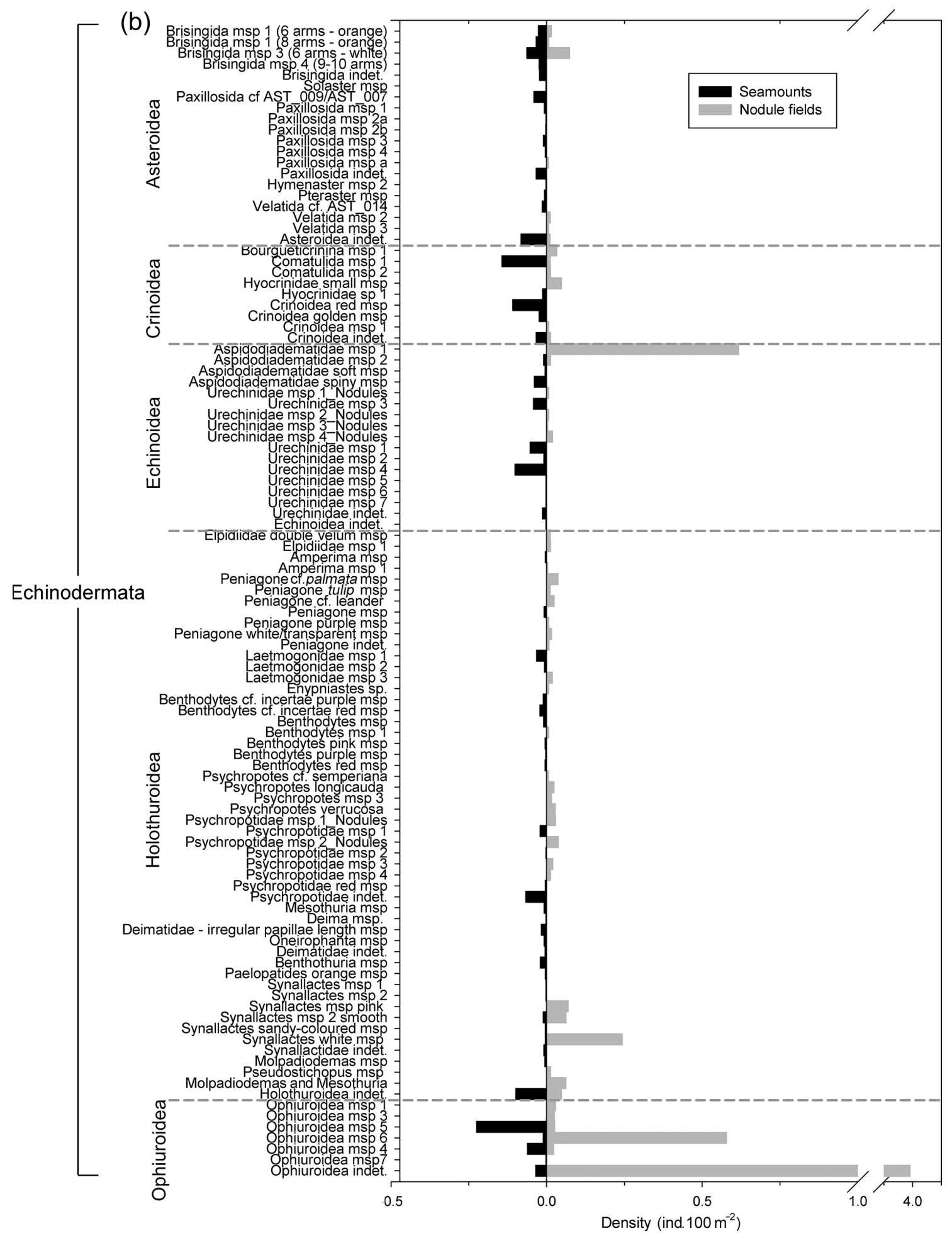

Figure A1. Continued. 
(c)

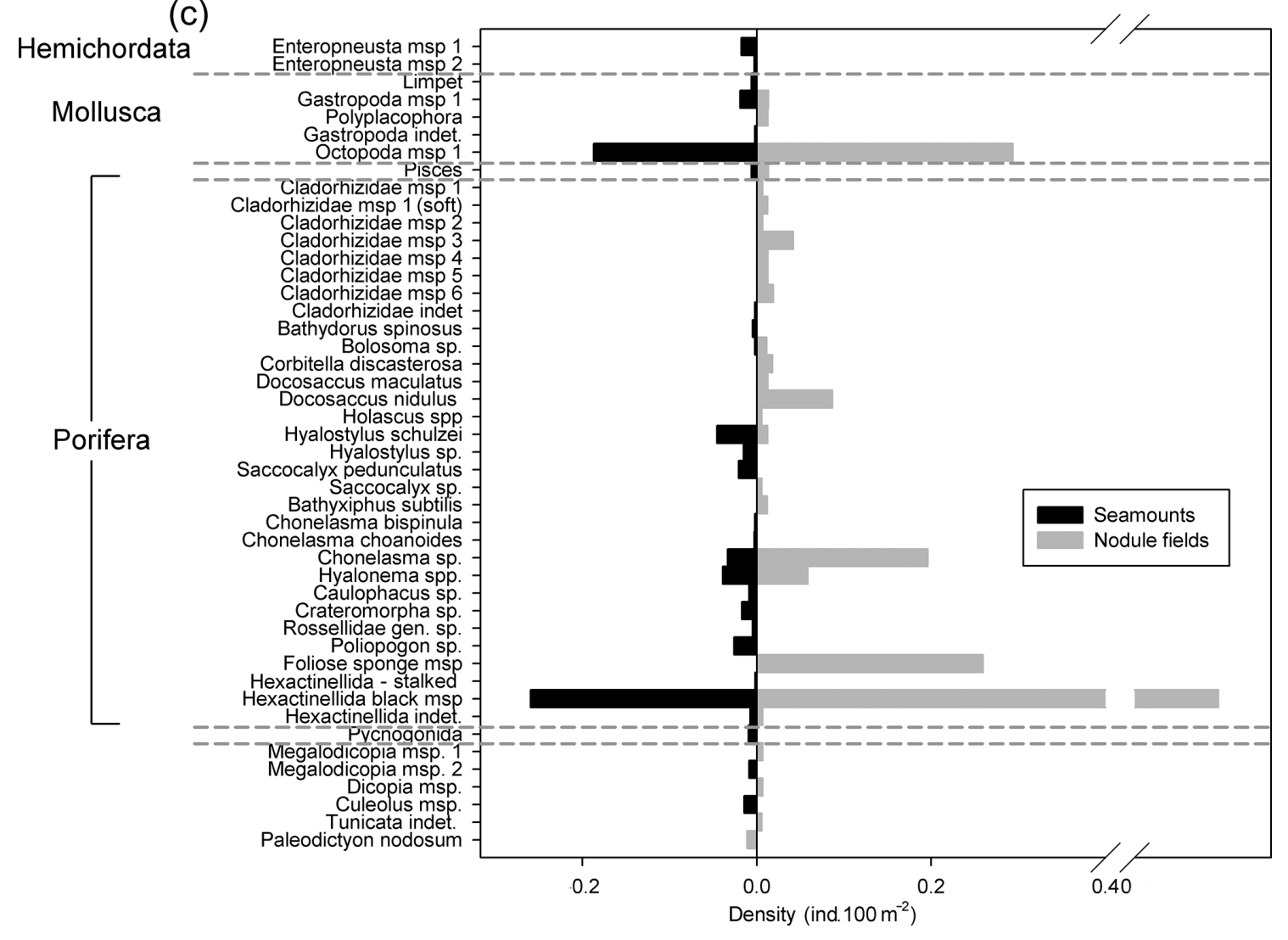

Figure A1. Continued. 
Data availability. Data sets are made available through OSIS-Kiel data portal, BIIGLE and PANGAEA.

Author contributions. DC, PAR, SPR and DK analysed the images. DC analysed the data. PMA, PAR and AC conceptualised and carried out the sampling. All authors contributed to the redaction of the manuscript.

Competing interests. The authors declare that they have no conflict of interest.

Special issue statement. This article is part of the special issue "Assessing environmental impacts of deep-sea mining - revisiting decade-old benthic disturbances in Pacific nodule areas". It is not associated with a conference.

Acknowledgements. We thank the crew of SO239 and GEOMAR for their support in acquiring the images used in this article. The EcoResponse cruise with RV Sonne was financed by the German Ministry of Education and Research (BMBF) as a contribution to the European project JPI Oceans, Ecological Aspects of Deep-Sea Mining. Pictures were provided by GEOMAR (Kiel).

Financial support. This study had the support of PO AÇORES 2020 project Acores-01-0145-Feder-000054_RECO and of Fundação para a Ciência e Tecnologia (Foundation for Science and Technology; FCT), through the strategic projects under UID/MAR/04292/2013 granted to MARE (Marine and Environmental Sciences Centre). The authors acknowledge funding from the JPI Oceans Ecological Aspects of Deep Sea Mining project by the FCT (Mining2/0005/2017) and the European Union Seventh Framework Programme (FP7/2007-2013) under the MIDAS project, grant agreement no. 603418. DC is supported by a postdoctoral scholarship (SFRH/BPD/110278/2015) from FCT. PAR was funded by FCT through a postdoctoral grant (ref. SFRH/BPD/69232/2010) funded through QREN and COMPETE. SPR is supported by FCT within the scope of the CEEC Individual 2017 contract (CEECIND/00758/2017) and by CESAM (UIDP/50017/2020CUIDB/50017/2020), through national funds from FCT under MCTES (the Portuguese Ministry of Science, Technology and Higher Education). AC is supported by the Investigador programme (IF/00029/2014/CP1230/CT0002) of FCT. PMA acknowledges funding from BMBF contract 03 F0707E.

Review statement. This paper was edited by Matthias Haeckel and reviewed by two anonymous referees.

\section{References}

Amon, D. J., Ziegler, A. F., Dahlgren, T. G., Glover, A. G., Goineau, A., Gooday, A. J., Wiklund, H., and Smith, C.
R.: Insights into the abundance and diversity of abyssal megafauna in a polymetallic-nodule region in the eastern Clarion-Clipperton Zone, Sci. Rep.-UK, 6, 30492, https://doi.org/10.1038/srep30492, 2016.

Baco, A. R.: Exploration for deep-sea corals on North Pacific seamounts and islands, Oceanography, 20, 109-117, 2007.

Beaulieu, S. E.: Colonization of habitat islands in the deep sea: Recruitment to glass sponge stalks, Deep-Sea Res. Pt. I, 48, 11211137, https://doi.org/10.1016/S0967-0637(00)00055-8, 2001.

Bluhm, H.: Re-establishment of an abyssal megabenthic community after experimental physical disturbance of the seafloor, DeepSea Res. Pt. II, 48, 3841-3868, https://doi.org/10.1016/S09670645(01)00070-4, 2001.

Bluhm, H. and Gebruk, A. V.: Holothuroidea (Echinodermata) of the Peru basin - ecological and taxonomic remarks based on underwater images, Mar. Ecol., 20, 167-195, https://doi.org/10.1046/j.1439-0485.1999.00072.x, 1999.

Boschen, R. E., Rowden, A. A., Clark, M. R., Barton, S., Pallentin, A., and Gardner, J.:Megabenthic assemblage structure on three New Zealand seamounts: implications for seafloor massive sulfide mining. Mar. Ecol. Prog. Ser., 523, 1-14, https://doi.org/10.3354/meps11239, 2015.

Clark, M. R., Rowden, A. A., Schlacher, T., Williams, A., Consalvey, M., Stocks, K. I., Rogers, A. D., O’Hara, T. D., White, M., Shank, T. M., and Hall-Spencer, J. M.: The Ecology of Seamounts: Structure, Function, and Human Impacts, Annu. Rev. Mar. Sci., 2, 253-278, https://doi.org/10.1146/annurevmarine-120308-081109, 2010

Clark, M. R., Schlacher, T. A., Rowden, A. A., K. Stocks, K. I., and Consalvey, M.: Science priorities for seamounts: research links to conservation and management, PLOS ONE, 7, e29232, https://doi.org/10.1371/journal.pone.0029232, 2012.

Durden, J. M., Bett, B. J., and Ruhl, H. A.: The hemisessile lifestyle and feeding strategies of Iosactis vagabunda (Actiniaria, Iosactiidae), a dominant megafaunal species of the Porcupine Abyssal Plain, Deep-Sea Res. Pt. I, 102, 72-77, https://doi.org/10.1016/j.dsr.2015.04.010, 2015a.

Durden, J. M., Bett, B. J., Jones, D. O. B., Huvenne, V. A. I., and Ruhl, H. A.: Abyssal hills - hidden source of increased habitat heterogeneity, benthic megafaunal biomass and diversity in the deep sea, Prog. Oceanogr., 137, 209-218, https://doi.org/10.1016/j.pocean.2015.06.006, 2015 b.

Genin, A., Dayton, P. K., Lonsdale, P., and Spiess, F. N.: Corals on seamount peaks provide evidence of current acceleration over deep-sea topography, Nature, 322, 59-61, 1986.

International Seabed Authority (ISA): https://www.isa.org.jm/ scientific-glossary/, last access: 29 October 2019.

Kamenskaya, O. E., Gooday, A. J., Tendal, O. S., and Melnik, V. F.: Xenophyophores (Protista, Foraminifera) from the ClarionClipperton Fracture Zone with description of three new species, Mar. Biodivers., 45, 581-593, https://doi.org/10.1007/s12526015-0330-z, 2015.

Kersken, D., Janussen, D., and Martinez Arbizu, P.: Deep-sea glass sponges (Hexactinellida) from polymetallic nodule fields in the Clarion-Clipperton Fracture Zone (CCFZ), northeastern Pacific: Part I - Amphidiscophora, Mar. Biodivers., 48, 545-573, https://doi.org/10.1007/s10750-017-3498-3, 2018a.

Kersken, D., Janussen, D., and Martinez Arbizu, P.: Deepsea glass sponges (Hexactinellida) from polymetallic nodule fields in 
the Clarion-Clipperton Fracture Zone (CCFZ), northeastern Pacific: Part II - Hexasterophora, Mar. Biodivers., 48, 545-573, https://doi.org/10.1007/s12526-018-0880-y, 2018b.

Kitchingman, A., Lai, S., Morato, T., and Pauly, D.: Seamount abundance and locations, in: Seamounts: Ecology, Fisheries and Conservation, edited by: Pitcher, T. J., Morato, T., Hart, P. J. B., Clark, M., Haggan, N., and Santos, R., Fish and Aquatic Resources Series, Blackwell, Oxford , UK, 26-40, 2007.

Lampitt, R. S. and Paterson, G. L. J.: The feeding behaviour of an abyssal sea anemone from in situ time lapse photographs and trawl samples, Oceanol. Acta, 10, 455-461, 1987.

Lapointe, L. and Bourget, E.: Influence of substratum heterogeneity scales and complexity on a temperate epibenthic marine community, Mar. Ecol. Prog. Ser., 189, 159-170, https://doi.org/10.3354/meps189159, 1999.

Leitner, A. B., Neuheimer, A. B., Donlon, E., Smith, C. R., and Drazen, J. C.: Environmental and bathymetric influences on abyssal bait-attending communities of the Clarion Clipperton Zone, Deep-Sea Res. Pt. I, 125, 65-80, https://doi.org/10.1016/j.dsr.2017.04.017, 2017.

Levin, L., DeMaster, D., McCann, L., and Thomas, C.: Effects of giant protozoans (class: Xenophyophorea) on deepseamount benthos, Mar. Ecol. Prog. Ser., 29, 99-104, https://doi.org/10.3354/meps029099, 1986.

McClain, C. R., Lundsten, L., Barry, J., and DeVogelaere, A.: Assemblage structure, but not diversity or density, change with depth on a northeast Pacific seamount, Mar. Ecol., 31, 14-25. https://doi.org/10.1111/j.1439-0485.2010.00367.x, 2010.

McClain, C. R., Lundsten, L., Ream, M., Barry, J., and DeVogelaere, A.: Endemicity, biogeography, composition, and community structure on a Northeast Pacific seamount, PLOS ONE, 4, e4141, https://doi.org/10.1371/journal.pone.0004141, 2009.

Mullineaux, L. S.: The role of settlement in structuring a hardsubstratum community in the deep sea, J. Exp. Mar. Biol. Ecol., 120, 241-261, 1988.

O'Hara, T. D.: Seamounts: Centres of endemism or species richness for ophiuroids? Glob. Ecol. Biogeogr., 16, 720-732, https://doi.org/10.1111/j.1466-8238.2007.00329.x, 2007.

Oksanen, J., Blanchet, G., Friendly, M., Kindt, R., Legendre, P., McGlinn, D., Minchin, P. R., O'Hara, R. B., Simpson, G. L., Solymos, P., Stevens, M. H. H., Szoecs, E., and Wagner, H.: vegan: Community Ecology Package, R package version 2.5-2, available at: https://CRAN.R-project.org/package=vegan, 2018.

Rappaport, Y., Naar, D. F., Barton, C. C., Liu, Z. J., and Hey, R. N.: Morphology and distribution of seamounts surrounding Easter Island, J. Geophys. Res., 102, 24713, https://doi.org/10.1029/97JB01634, 1997.

Raymore, P. A.: Photographic investigations on three seamounts in the Gulf of Alaska, Pac. Sci., 36, 15-34, 1982.
R Core Team: R: A language and environment for statistical computing. R Foundation for Statistical Computing, Vienna, Austria, available at: https://www.R-project.org/, 2018.

Riemann-Zürneck, K.: How sessile are sea anemones? A review of free-living forms in the Actiniaria (Cnidaria: Anthozoa), Mar. Ecol., 19, 247-261, https://doi.org/10.1111/j.14390485.1998.tb00466.x, 1998.

Rogers, A. D.: The Biology of Seamounts: 25 years on, Adv. Mar. Biol., 79, 137-224, https://doi.org/10.1016/bs.amb.2018.06.001, 2018.

Rowden, A. A., Schlacher, T. A., Williams, A., Clark, M. R., Stewart, R., Althaus, F., Bowden, D. A., Consalvey, M. Robinson, W., and Dowdney, J.: A test of the seamount oasis hypothesis: seamounts support higher epibenthic megafaunal biomass than adjacent slopes, Mar. Ecol., 31, 95-106, https://doi.org/10.1111/j.1439-0485.2010.00369.x, 2010.

Simon-lledó, E., Bett, B. J., Huvenne, V. A. I., Schoening, T., Benoist, N. M. A., Je, R. M., Durden, J. M., and Jones, D. O. B.: Megafaunal variation in the abyssal landscape of the Clarion Clipperton Zone, Progr. Oceanogr. 170, 119-133, https://doi.org/10.1016/j.pocean.2018.11.003, 2019.

Stoyanova, V.: Megafaunal diversity associated with deep-sea nodule-bearing habitats in the eastern part of the clarionclipperton zone, NE pacific. Int. Multidiscipl. Sci., 1, https://doi.org/10.5593/sgem2012/s03.v1032, 2012.

Tilot, V., Ormond, R., Moreno Navas, J., and Catalá, T. S.: The Benthic Megafaunal Assemblages of the CCZ (Eastern Pacific) and an Approach to their Management in the Face of Threatened Anthropogenic Impacts, Front. Mar. Sci., 5, 1-25, https://doi.org/10.3389/fmars.2018.00007, 2018.

Vanreusel, A., Hilario, A., Ribeiro, P. A., Menot, L., and Arbizu, P. M.: Threatened by mining, polymetallic nodules are required to preserve abyssal epifauna, Sci. Rep.-UK, 6, 26808, https://doi.org/10.1038/srep26808, 2016.

Volz, J. B., Mogollón, J. M., Geibert, W., Arbizu, P. M., Koschinsky, A., and Kasten, S.: Natural spatial variability of depositional conditions, biogeochemical processes and element fluxes in sediments of the eastern Clarion-Clipperton Zone, Pacific Ocean, Deep Sea Res. Pt. I, 140, 159-172, https://doi.org/10.1016/j.dsr.2018.08.006, 2018.

Wedding, L. M., Friedlander, A. M., Kittinger, J. N., Watling, L., Gaines, S. D., Bennett, M., Hardy, S. M., and Smith, C. R.: From principles to practice?: a spatial approach to systematic conservation planning in the deep sea, P. Roy. Soc. B-Biol. Sci., 280 20131684, https://doi.org/10.1098/rspb.2013.1684, 2013.

Wessel, P., Sandwell, D., and Kim, S.-S.: The Global Seamount Census, Oceanography, 23, 24-33, https://doi.org/10.5670/oceanog.2010.60, 2010. 